\title{
Die Wahl Obamas zum 44. Präsidenten der USA
}

\section{Kurzfassung}

Mit Barack Obama wurde 143 Jahre nach Abschaffung der Sklaverei erstmals ein Schwarzer in das Präsidentenamt der USA gewählt. Der historische Wahlerfolg des demokratischen Senators aus Illinois kam nicht überraschend; das Ergebnis war in erster Linie ein Referendum gegen den unpopulären Präsidenten George W. Bush, der für eine katastrophale Wirtschaftslage und einen umstrittenen Krieg im Irak verantwortlich zeichnete. John McCain konnte sich von dem negativen Image Bushs und seiner Partei nicht absetzen. Als maverick entfachte er lange Zeit kaum Enthusiasmus bei der sozialkonservativen Parteibasis und warb zu wenig Wahlkampfspenden ein. Im Versuch das Steuer herumzureißen kam er den Positionen Bushs inhaltlich weit entgegen und beging mit der Nominierung der unerfahrenen Sarah Palin zur Vizepräsidentschaftskandidatin einen strategischen Fehler. Der durch die Rahmenbedingungen dieser Wahl favorisierte Obama profitierte in der Endphase des Wahlkampfs von den Hiobsbotschaften vom Finanzmarkt, die alles Augenmerk auf das wahlentscheidende Wirtschaftsthema lenkten. Sein ruhiges, selbstsicheres Auftreten in den TV-Duellen flößte Vertrauen ein und zerstreute letzte Zweifel an seiner Eignung fürs Amt. Ein rassisch motiverter Wählerumschwung weißer Wähler zugunsten McCains blieb auf wenige Staaten im tiefen Süden beschränkt und wurde durch den Zulauf junger, schwarzer und hispanischer Wähler ins Lager Obamas kompensiert. Seinen eigentlichen Sieg hatte Obama in der demokratischen Primary gegen Hillary Clinton errungen. Hier legte er - auch organisatorisch - die Grundsteine für seinen späteren Wahlerfolg. 


\section{Inhalt}

1. Einleitung 108

2. Die Wahlergebnisse im Überblick 109

3. Die Geografie des Sieges 111

4. Die Soziografie des Sieges 115

5. Die Motive der Wahlentscheidung: Themen und Kandidaten 122

6. Der Beitrag der Wahlkämpfe zum Erfolg Obamas 128

a) Der Wahlkampf McCains 129

b) Der Wahlkampf Obamas 133

7. Ausblick: Vom Wahlkampf zum Regieren 139

\section{Einleitung}

„I was never the likeliest candidate for this office. We didn't start with much money or many endorsements. Our campaign was not hatched in the halls of Washington. It began in the backyards of Des Moines and the living rooms of Concord and the front porches of Charleston. It was built by working men and women who dug into what little savings they had to give 5 dollars, 10 dollars and 20 dollars to the cause. It grew strength from the young people who rejected the myth of their generation's apathy, who left their homes and their families for jobs that offered little pay and less sleep. It drew strength from the not-so-young people who braved the bitter cold and scorching heat to knock on doors of perfect strangers, and from the millions of Americans who volunteered and organized and proved that more than two centuries later a government of the people, by the people, and for the people has not perished from the Earth. This is your victory." (Barack Obama, Chicago, Illinois, 4. November 2008)

Amerikaner lieben die underdogs, die Außenseiter, die schließlich doch alle Widrigkeiten und Hindernisse überwinden, um zum strahlenden Helden aufzusteigen. Barack Obama erkannte schon früh sein biografisches Potenzial, den Amerikanischen Traum zu verkörpern (Obama 2004, 2006) - doch war er deshalb „nie der wahrscheinlichste Kandidat“ für das Präsidentenamt, wie er es selbst am Abend seines historischen Sieges in Chicago gesagt hat? An 
einer solchen Darstellung sind Zweifel angebracht: Nach allen Grundlagenerkenntnissen der Wahlforschung musste der demokratische Kandidat in dieser Präsidentschaftswahl als der klare Favorit gelten. Ein über alle Maßen unbeliebter Präsident, der für eine katastrophale Wirtschaftslage und einen zunehmend unpopulären Krieg im Irak verantwortlich zeichnete, hinterließ dem Kandidaten der Republikanischen Partei ein Erbe, das ihn unter normalen Umständen zum Verlierer dieser Wahl machen musste. Die Frage konnte nur sein, ob außergewöhnliche Ereignisse - ein Terroranschlag, grobe Fehler im Wahlkampf oder ein rassistisch motivierter Wählerumschwung in der Wahl - den Sieg Obamas noch in letzter Minute würden verhindern können.

Dennoch hat Obama mit seiner Sichtweise auf den Sieg - aus der Binnenperspektive des Wahlkämpfers - nicht Unrecht. Es gibt keine Determinismen in der Politik, schon gar keine automatischen Wahlsiege, die sich ohne weiteres Zutun der Kandidaten einstellen. Obama mochte aufgrund der objektiven Rahmenbedingungen der klare Favorit dieser Wahl sein, deswegen hatte er sie aber noch lange nicht gewonnen. Vorsprünge lassen sich verspielen - nirgends dürfte diese Sportlerweisheit größere Gültigkeit beanspruchen als in dem wohl brutalsten Ausscheidungswettlauf um ein politisches Amt, den die westliche Welt kennt. Dies gilt erst recht, wenn neben dem Hauptwahlkampf auch die Vorwahlen einschließlich ihres Vorlaufs Berücksichtigung finden. Wer diesen zweijährigen Wettbewerb erfolgreich absolviert, ist immer ein unwahrscheinlicher Sieger - auch wenn er wie Barack Obama der Favorit in der Hauptwahl und nach Hillary Clinton der zweite Anwärter auf den Sieg in der demokratischen Vorwahl war (Cohen u. a. 2008a; Walker 2008).

\section{Die Wahlergebnisse im Überblick}

Der Sieger der Präsidentschaftswahl stand diesmal schon früh am Abend des 4. November 2008 fest: Barack Obama, erst im vierten Jahr Senator von Illinois, hatte knapp 70 Millionen Amerikaner zur Stimmabgabe für ihn bewegt, fast elf Millionen mehr als John F. Kerry bei der vorherigen Präsidentschaftswahl. Obamas Konkurrent John McCain hatte dagegen nur 60 Millionen Stimmen hinter sich gebracht, etwa zwei Millionen weniger als George W. Bush 2004. Der Vorsprung Obamas im popular vote fiel mit $53 \mathrm{zu} 46 \%$ so deutlich aus, wie es die Wahlabsichtsumfragen seit Ende September durchweg ermittelt hatten. Die von manchen befürchtete Überraschung eines rassisch motivierten Wählerumschwungs in den letzten Tagen vor der Wahl trat nicht ein. 
Tabelle 1

Die Ergebnisse der Wahlen vom 4. November 2008 mit nationaler Ausstrahlung im Vergleich zur vorherigen Wahl

\begin{tabular}{|c|c|c|c|c|c|c|}
\hline Präsidentschafts- & \multicolumn{3}{|c|}{2008} & \multicolumn{3}{|c|}{2004} \\
\hline Kandidat & Obama & McCain & Insges. & Kerry & Bush & Insges. \\
\hline $\begin{array}{l}\text { Popular Vote } \\
\text { (in Mio.) }\end{array}$ & 69,5 & 59,9 & 131,2 & 58,9 & 61,9 & 122,3 \\
\hline PV (in \%) & 53,0 & 45,7 & 100,0 & 48,1 & 50,6 & 100,0 \\
\hline Electoral Vote & 365 & 173 & 538 & 251 & 286 & 538 \\
\hline EV (in \%) & 67,8 & 32,2 & 100,0 & 46,7 & 53,2 & 100,0 \\
\hline VAP $^{a}$ (in Mio.) & - & - & 231,2 & - & - & 220,8 \\
\hline VAP Turnout (in \%) & - & - & 56,8 & - & - & 55,4 \\
\hline $\mathrm{VEP}^{\mathrm{b}}$ (in Mio.) & - & - & 213,0 & - & - & 203,5 \\
\hline VEP Turnout (in \%) & - & - & 61,6 & - & - & 60,1 \\
\hline Kongresswahlen & \multicolumn{3}{|c|}{2008} & \multicolumn{3}{|c|}{ Vor der Wahl } \\
\hline & $\mathrm{D}$ & $\mathrm{R}$ & $\mathrm{I}$ & $\mathrm{D}$ & $\mathrm{R}$ & I \\
\hline Senatoren $^{c}$ & 56 & 41 & $2^{\mathrm{d}}$ & 49 & 49 & $2^{\mathrm{d}}$ \\
\hline Repräsentanten & 257 & 178 & - & 236 & 199 & - \\
\hline $\begin{array}{l}\text { Einzelstaatl. } \\
\text { Wahlen }\end{array}$ & \multicolumn{3}{|c|}{2008} & \multicolumn{3}{|c|}{ Vor der Wahl } \\
\hline Gouverneure & 29 & 21 & - & 28 & 22 & - \\
\hline & $\mathrm{D}$ & $\mathrm{R}$ & Geteilt & $\mathrm{D}$ & $\mathrm{R}$ & Geteilt \\
\hline $\begin{array}{l}\text { Kontrolle } \\
\text { Legislativen }\end{array}$ & 27 & 14 & 8 & 22 & 15 & 12 \\
\hline
\end{tabular}

${ }^{\mathrm{a}}$ Voting-Age Population (geschätzt). ${ }^{\mathrm{b}}$ Voting-Eligible Population (geschätzt). ${ }^{\mathrm{c} U ̈ b e r}$ einen Senatssitz in Minnesota wird noch in einer Nachzählung entschieden. ${ }^{\mathrm{d} D i e}$ beiden unabhängigen Senatoren Joe Lieberman (Connecticut) und Bernie Sanders (Vermont) haben sich in der Fraktion der Demokraten organisiert. Quellen: CNN 2008; McDonald 2008; NCSL 2008; Storey/Smith 2008: 15; U.S. Census 2008: 241, 249.

Am Ende reichte es für den ersten schwarzen Präsidenten in der Geschichte der USA zu einer Mehrheit von 365 zu 173 Stimmen im Electoral College. Dies war zwar kein Erdrutschsieg, aber ein deutliches „Mandat“ für 
den Wechsel ${ }^{1}$ - zumal Obama auch signifikante presidential coattails mit sich zog: Im Senat bauten die Demokraten ihre Mehrheit um sieben auf nunmehr 58 zu 41 Sitze aus (ein Rennen ist noch offen); im Repräsentantenhaus verhalf Obama seiner Partei zu 21 neuen Abgeordneten: die Mehrheit beträgt dort nun 257 zu 178 Sitze. Einen größeren presidential coattail gab es zuletzt 1980 bei der Wahl Ronald Reagans zum Präsidenten, als die Republikaner 12 Sitze im Senat und 35 im Repräsentantenhaus hinzugewannen (Davidson/ Oleszek 2006: Appendix A). Auch bei den Wahlen in den Einzelstaaten, zum Gouverneur und zu den einzelstaatlichen Legislativen, schnitten die Demokraten erfolgreich ab: Sie erhöhten die Zahl ihrer Gouverneure von 28 auf 29 und die Zahl der Einzelstaaten, in denen sie beide Kammern der Legislativen kontrollierten, von 22 auf 27. Die Wahlbeteiligung stieg nach 2000 und 2004 zum dritten Mal in Folge an und erreichte mit geschätzten knapp $62 \%$ ihren höchsten Wert seit 1968 (Tabelle 1).

\section{Die Geografie des Sieges}

Obama gewann alle Staaten im Nordosten der USA - und damit auch alle dort zu vergebenden 101 Elektorenstimmen. Der Nordosten ist in den letzten Jahrzehnten zur fast uneinnehmbaren Hochburg der Demokraten geworden; George H. W. Bush war 1988 der letzte republikanische Präsidentschaftskandidat, der eine Mehrheit der Elektorenstimmen aus dem Nordosten hinter sich bringen konnte. John F. Kerry gewann hier 2004 alle Staaten, Al Gore verlor im Jahre 2000 nur New Hampshire an George W. Bush. Im Vorfeld der Wahl hatten einzig New Hampshire und Pennsylvania zeitweilig als Staaten mit Außenseiterchancen für John McCain gegolten. Am Ende gewann Obama beide Staaten mit etwa zehn Prozentpunkten Vorsprung. Wie fest der Nordosten mittlerweile in demokratischer Hand ist, lässt sich auch daran ablesen, dass aus

1 Mandate inhaltlicher Art lassen sich aus Präsidentschaftswahlergebnissen objektiv nur schwer ableiten; dennoch sind Wahlsieger naturgemäß versucht, ihr Mandat programmatisch zu definieren, um ihre Erfolgsaussichten im Kongress zu erhöhen. Wenn sie ihr Mandat überdehnen - wie Präsident Bush nach seiner Wiederwahl 2004 -, können sie aber Schiffbruch erleiden (Edwards 2007: 211-246). Im Grunde verfügt jeder neue Präsident, so formulieren es Polsby/Wildavsky (2008: 261), über das Mandat, „welche policies auch immer zu verfolgen, die ihm oder ihr gefallen“. Konkrete Mandate programmatischer Art - wie bei Roosevelt 1932 (New Deal), Johnson 1964 (Great Society) oder Reagan 1980 (Beschneidung des big government) - gibt es aber nur, wenn es einem Präsidenten gelingt, Mehrheiten in Kongress und Öffentlichkeit davon zu überzeugen, dass er tatsächlich mit einem solchen Mandat vom Wähler ausgestattet wurde. 
den Neuenglandstaaten (Maine, New Hampshire, Vermont, Massachusetts, Rhode Island und Connecticut) 2008 kein einziger Republikaner mehr in den Kongress gewählt worden ist. Auf einzelstaatlicher Ebene gibt es nach dieser Wahl im gesamten Nordosten nur noch eine einzige Kammer der Legislative, die von den Republikanern kontrolliert wird: der Senat von Pennsylvania (Storey/Smith 2008: 14).

Tabelle 2

Das Präsidentschaftswahlergebnis 2008 nach Regionen, Electoral Vote

\begin{tabular}{|c|c|c|}
\hline & Obama 365 EV & McCain 173 EV \\
\hline $\begin{array}{l}\text { Westen } \\
\text { (124 EV) }\end{array}$ & $\begin{array}{l}\text { California 55, Colorado 9, } \\
\text { Hawaii 4, Nevada 5, New } \\
\text { Mexico 5, Oregon 7, } \\
\text { Washington } 11=96 \mathrm{EV} \\
\end{array}$ & $\begin{array}{l}\text { Alaska 3, Arizona 10, Idaho 4, } \\
\text { Montana 3, Utah 5, Wyoming } 3 \\
=28 \mathrm{EV}\end{array}$ \\
\hline $\begin{array}{l}\text { Mitlerer } \\
\text { Westen } \\
\text { (124 EV) }\end{array}$ & $\begin{array}{l}\text { Michigan 17, Minnesota 10, } \\
\text { Illinois 21, Indiana 11, Iowa 7, } \\
\text { Nebraska 1, Ohio 20, } \\
\text { Wisconsin } 10=97 \mathrm{EV}\end{array}$ & $\begin{array}{l}\text { Kansas 6, Missouri 11, Nebraska } \\
\text { 4, North Dakota 3, South Dakota } \\
3=27 \mathrm{EV}\end{array}$ \\
\hline \begin{tabular}{|l} 
Süden \\
$(189 \mathrm{EV})$
\end{tabular} & $\begin{array}{l}\text { Delaware 3, District of } \\
\text { Columbia 3, Florida 27, } \\
\text { Maryland 10, North Carolina } \\
\text { 15, Virginia } 13=71 \mathrm{EV}\end{array}$ & $\begin{array}{l}\text { Alabama 9, Arkansas 6, Georgia } \\
\text { 15, Kentucky 8, Louisiana 9, } \\
\text { Mississippi 6, Oklahoma 7, South } \\
\text { Carolina 8, Tennessee 11, Texas } \\
\text { 34, West Virginia 5 = 118 EV }\end{array}$ \\
\hline \begin{tabular}{|l|} 
Nord- \\
osten \\
$(101$ EV)
\end{tabular} & $\begin{array}{l}\text { Connecticut 7, Maine 4, Massa- } \\
\text { chusetts 12, New Hampshire 4, } \\
\text { New Jersey 15, New York 31, } \\
\text { Pennsylvania 21, Rhode Island } \\
\text { 4, Vermont 3 = 101 EV }\end{array}$ & \\
\hline
\end{tabular}

Quelle: New York Times (Stand: 21.11.08).

Außer im Nordosten hatte Obama vor allem im Westen ein Heimspiel, insbesondere in den Staaten an der Pazifikküste. Seit 1992 hatten alle demokratischen Präsidentschaftskandidaten Washington, Oregon, Kalifornien und Hawaii gewonnen. Es konnte deshalb nicht überraschen, dass auch Obama diese Staaten eroberte: Kalifornien mit 61, Hawaii, die Heimstätte seiner Kindheit und seiner kurz vor der Wahl verstorbenen Großmutter, mit $72 \%$ 
der abgegebenen Stimmen. Obama siegte darüber hinaus in den battleground states Nevada, Colorado und New Mexico - Staaten, die 2004 noch George W. Bush gewonnen hatte. McCain konnte seinen Heimatstaat Arizona, den Heimatstaat seiner Vizepräsidentschaftskandidatin Sarah Palin, Alaska, sowie die an Bevölkerung armen und an Bodenschätzen reichen Staaten im Nordwesten hinter sich bringen: Idaho, den Mormonenstaat Utah, die Great Plains-Staaten Montana und Wyoming (Tabelle 2).

Die meisten battleground states lagen 2008 wieder im Mittleren Westen: Ohio mit 20, Missouri mit elf und Iowa mit sieben Elektorenstimmen waren wie eh und je die am härtesten umkämpften Staaten. Traditionell eher den Demokraten zuneigend, zählten 2008 auch Minnesota, Wisconsin und Michigan wieder zu den stark von beiden Parteien umworbenen Staaten. Mit Ausnahme von Missouri gewann Obama alle battleground states im Mittleren Westen. Da er auch seinen Heimatstaat Illinois und den Nachbarstaat Indiana, traditionell eine Hochburg der Republikaner, für die Demokraten eroberte, färbte sich die Wahl-Landkarte um das Gebiet der Großen Seen komplett blau. Für die von McCain angeführten Republikaner blieben nur die bevölkerungsschwachen, stark landwirtschaftlich geprägten Prärie-Staaten North Dakota, South Dakota, Nebraska und Kansas. In Nebraska, das neben Maine als einziger Bundesstaat seine Elektorenstimmen nach dem Distrikt- und nicht nach dem Winner-Takes-All-System zuweist (Turner 2005), gewann Obama einen Kongressdistrikt hauchdünn vor McCain und somit eines der fünf electoral votes des Staates (Tabelle 2).

In historischer Hinsicht das bemerkenswerteste Ergebnis der Präsidentschaftswahl 2008 war die Tatsache, dass Obama sich auch im Süden als wettbewerbsfähig erwies. Seit 1968 bei Präsidentschaftswahlen zu einer ausgesprochenen Hochburg der Republikaner geworden (Black/Black 2002), galt im Prinzip der Erfahrungssatz, dass im Süden nur ein gemäßigter demokratischer Präsidentschaftskandidat aus der Region selbst auf Augenhöhe mit den Republikanern konkurrieren könne. Obama kam aus der Demokraten-Hochburg Chicago (Illinois), er hatte den progressivsten voting record im Senat, er war schwarz - und er gewann dennoch 71 von 189 Elektorenstimmen des Südens. Wenig überraschend waren seine Siege in der Bundeshauptstadt Washington, D.C., im ökonomisch prosperierenden Delaware, der Heimat von Vizepräsidentschaftskandidat Joe Biden, und in Maryland - alles Staaten, die politisch, kulturell und ökonomisch inzwischen eher dem Nordosten als dem Süden zuzurechnen sind. Auch der Sieg im battleground state Florida war erwartet worden. Mit Virginia und North Carolina gelangen Obama jedoch 
Einbrüche in den Upper South, die historisch ganz und gar unwahrscheinlich erschienen: North Carolina hatte zuletzt 1976 (Jimmy Carter), Virginia 1964 (Lyndon B. Johnson) für einen demokratischen Präsidentschaftskandidaten gestimmt. Nur der Deep South mit Ausnahme Floridas - also South Carolina, Georgia, Alabama, Mississippi, Arkansas, Louisiana und Texas - verblieb auch 2008 fest in republikanischer Hand. Den Great Plains-Staat Oklahoma, die beiden Upper South-Staaten Tennessee und Kentucky sowie das Armenhaus West Virginia konnte McCain ebenfalls für die Republikaner halten (Tabelle 2).

Insgesamt gewann Obama in 28 Einzelstaaten und der Bundeshauptstadt Washington, D.C, McCain in 22 Einzelstaaten. Obama gewann eher bzw. höher in bevölkerungsreichen als in bevölkerungsarmen Staaten; er gewann eher bzw. höher an der Küste als im Landesinnern; er gewann eher bzw. höher in der Stadt als auf dem Lande; und er gewann eher bzw. höher in ökonomisch fortschrittlichen als rückständigen Regionen. Dies hatte auch 2008 wieder eine ,polarisierte“ Wahl-Landkarte zur Folge (Fiorina/Abrams/Pope 2005), die sich im Nordosten (plus den Küstenstaaten des Oberen Südens), um das Gebiet der Großen Seen herum, in Florida sowie im äußersten Westen der USA demokratisch blau färbte, während das Landesinnere - mit Ausnahme Colorados und New Mexicos - dem Rot der Republikaner vorbehalten blieb.

Wie geografisch ,polarisiert“ bzw. parteilich homogen die Amerikaner 2008 wieder wählten, lässt sich auch an ihrem Verhalten in den Kongresswahlen und den einzelstaatlichen Wahlen ablesen: Alle Zugewinne der Demokraten im Kongress (in Colorado, New Mexico und Oregon im Westen, in Illinois, Michigan und Ohio im Mittleren Westen, in New Hampshire und New York im Nordosten sowie in North Carolina und Virginia im Süden) fanden in Staaten statt, die Obama gewonnen hatte, waren mithin echte presidential coattails. Gleiches galt für die Eroberung der Mehrheit in den fünf einzelstaatlichen Legislativkammern in Delaware, Nevada, New York, Ohio und Wisconsin durch die Demokraten (NCSL 2008). Dies sind Indizien dafür, dass das split ticket voting wie schon 2000 und 2004 weiter zugunsten des party line voting zurücktrat - eine Trendumkehr im Wahlverhalten, die das in den Nachkriegsjahren dominierende divided government unwahrscheinlicher werden lässt (Jacobson 1990, 2007a: 197 ff.). 


\section{Die Soziografie des Sieges}

Obama wurde bei seinem Wahlsieg 2008 im Allgemeinen von denjenigen Gesellschaftsgruppen getragen, die schon immer bevorzugt die Demokraten gewählt haben - nur dass sie es diesmal in der Regel noch ein wenig mehr als in der Vergangenheit taten. Der in diesem Wahlkampf vielfach beschworene Enthusiasmus der Anhänger Obamas wirkte sich nicht nur in der demokratischen Vorwahl, sondern auch in der Hauptwahl zu seinen Gunsten aus. Denn auch wenn die Wahlbeteiligung insgesamt stieg, so waren die Zuwächse über die Parteianhängerschaften und die einzelnen Bevölkerungsgruppen hinweg doch ungleich verteilt: Vor allem die Jüngeren, die Schwarzen und die Latinos steigerten ihre Wahlbeteiligung diesmal signifikant. Bei den Schwarzen und mehr noch bei den Latinos, der am schnellsten wachsenden Bevölkerungsgruppe in den USA (Barnes/Cohen 2004; Greenblatt 2008), spiegelte sich in ihrem gewachsenen Anteil an der Wählerschaft auch der demografische Wandel wider. Dennoch mussten sich viele Angehörige dieser traditionell beteiligungsschwachen Gruppen als Wähler erst einmal registrieren lassen - und sie taten es fast ausschließlich aufseiten der Demokraten (Saslow 2008). Den Republikanern gelang dagegen die Mobilisierung ihrer Stammwähler - der Weißen, der Älteren und der protestantischen Kirchgänger nicht so gut wie noch 2004. Insgesamt identifizierten sich bei dieser Wahl $39 \%$ der Wähler als Demokraten, nur $32 \%$ als Republikaner (Tabelle 3).

Nach Abzug des enthusiasm gap zugunsten Obamas folgte das Wahlverhalten $2008 \mathrm{im}$ Wesentlichen vertrauten Bahnen: Wie die demokratischen Präsidentschaftskandidaten der letzten Jahre profitierte auch Obama vom gender gap im Wahlverhalten und fand überproportionalen Zuspruch beim weiblichen Geschlecht: 56 \% der Frauen wählten ihn, nur $43 \%$ McCain. Von den Männern wählten dagegen fast genauso viele McCain wie Obama (Tabelle 3). 
Tabelle 3

Wahlverhalten in den Präsidentschaftswahlen 2008, 2004 und 2000 laut Voter Exit Polls

\begin{tabular}{|c|c|c|c|c|c|c|c|c|c|}
\hline \multirow[t]{2}{*}{ Wählergruppe } & \multicolumn{3}{|c|}{ Wähleranteil } & \multicolumn{3}{|c|}{ McCain/Bush } & \multicolumn{3}{|c|}{$\begin{array}{c}\text { Obama/Kerry/ } \\
\text { Gore }\end{array}$} \\
\hline & 08 & 04 & 00 & 08 & 04 & 00 & 08 & 04 & 00 \\
\hline $\begin{array}{l}\text { Geschlecht } \\
\text { Männer }\end{array}$ & 47 & 46 & 48 & 48 & 55 & 53 & 49 & 44 & 42 \\
\hline Frauen & 53 & 54 & 52 & 43 & 48 & 43 & 56 & 51 & 54 \\
\hline Ethnie & & & & & & & & & \\
\hline Weiße & 74 & 77 & 81 & 55 & 58 & 54 & 43 & 41 & 42 \\
\hline Schwarze & 13 & 11 & 10 & 4 & 11 & 9 & 95 & 88 & 90 \\
\hline Latinos & 9 & 8 & 7 & 31 & 44 & 35 & 67 & 53 & 62 \\
\hline Asiaten & 2 & 2 & 2 & 35 & 44 & 41 & 62 & 56 & 55 \\
\hline Weiße Männer & 36 & 36 & 39 & 57 & 61 & 60 & 41 & 38 & 36 \\
\hline Weiße Frauen & 39 & 41 & 43 & 53 & 55 & 49 & 46 & 44 & 48 \\
\hline Schwarze Männer & 5 & 5 & 4 & 5 & 13 & 12 & 95 & 86 & 85 \\
\hline Schwarze Frauen & 7 & 7 & 6 & 3 & 10 & 6 & 96 & 90 & 94 \\
\hline Erstwähler & 11 & 11 & 9 & 30 & 46 & 43 & 69 & 53 & 52 \\
\hline Alter & & & & & & & & & \\
\hline 18-29-Jährige & 18 & 17 & 17 & 32 & 45 & 46 & 66 & 54 & 48 \\
\hline 30-44-Jährige & 29 & 29 & 33 & 46 & 53 & 49 & 52 & 46 & 48 \\
\hline 45-59-Jährige & $37^{\mathrm{a}}$ & 30 & 28 & $49^{\mathrm{a}}$ & 51 & 49 & $50^{\mathrm{a}}$ & 48 & 48 \\
\hline über 65-Jährige & 16 & 16 & 14 & 53 & 52 & 47 & 45 & 47 & 50 \\
\hline Status & & & & & & & & & \\
\hline Verheiratet & 66 & 63 & 65 & 52 & 57 & 53 & 47 & 42 & 44 \\
\hline Nicht verheiratet & 34 & 37 & 35 & 33 & 40 & 38 & 65 & 58 & 57 \\
\hline Kinder unter 18 Jahre & 40 & 37 & 39 & 45 & 53 & 52 & 53 & 45 & 45 \\
\hline Homo- oder bisexuell & 4 & 4 & 4 & 27 & 23 & 25 & 70 & 77 & 70 \\
\hline
\end{tabular}


Wahl Barack Obamas

\begin{tabular}{|c|c|c|c|c|c|c|c|c|c|}
\hline \multirow[t]{2}{*}{ Wählergruppe } & \multicolumn{3}{|c|}{ Wähleranteil } & \multicolumn{3}{|c|}{ McCain/Bush } & \multicolumn{3}{|c|}{$\begin{array}{c}\text { Obama/Kerry/ } \\
\text { Gore }\end{array}$} \\
\hline & 08 & 04 & 00 & 08 & 04 & 00 & 08 & 04 & 00 \\
\hline $\begin{array}{l}\text { Familieneinkommen } \\
<15.000\end{array}$ & 6 & 8 & 7 & 25 & 36 & 37 & 73 & 63 & 57 \\
\hline $15-29.999 \$$ & 12 & 15 & 16 & 37 & 42 & 41 & 60 & 57 & 54 \\
\hline $30-49.999 \$$ & 19 & 22 & 24 & 43 & 49 & 48 & 55 & 50 & 49 \\
\hline $50-74.999 \$$ & 21 & 23 & 25 & 49 & 56 & 51 & 48 & 43 & 46 \\
\hline $75-99.999 \$$ & 15 & 14 & 13 & 48 & 55 & 52 & 51 & 45 & 45 \\
\hline $100-149.999 \$$ & 14 & 11 & 15 & 51 & 57 & 54 & 48 & 42 & 43 \\
\hline $150-199.999 \$$ & 6 & 4 & 15 & 50 & 58 & 54 & 48 & 42 & 43 \\
\hline$>200.000 \$$ & 6 & 3 & 15 & 46 & 63 & 54 & 52 & 35 & 43 \\
\hline Bildung & & & & & & & & & \\
\hline High-School-Besuch & 4 & 4 & 5 & 35 & 49 & 39 & 63 & 50 & 59 \\
\hline High-School-Abschluss & 20 & 22 & 21 & 46 & 52 & 49 & 52 & 47 & 48 \\
\hline College ohne Abschluss & 31 & 32 & 32 & 47 & 54 & 51 & 51 & 46 & 45 \\
\hline College-Abschluss & 28 & 26 & 24 & 48 & 52 & 51 & 50 & 46 & 45 \\
\hline Postgraduierten- & & & & & & & & & \\
\hline Abschluss & 17 & 16 & 18 & 40 & 44 & 44 & 58 & 55 & 52 \\
\hline Religion & & & & & & & & & \\
\hline Protestanten & 54 & 54 & 54 & 54 & 59 & 56 & 45 & 40 & 42 \\
\hline Weiße Protestanten & 42 & 41 & 47 & 65 & 67 & 63 & 34 & 32 & 34 \\
\hline Weiße Wiedergeborene & 26 & 23 & 14 & 74 & 78 & 80 & 24 & 21 & 18 \\
\hline Katholiken & 27 & 27 & 26 & 45 & 52 & 47 & 54 & 47 & 50 \\
\hline Weiße Katholiken & 19 & 20 & 20 & 52 & 56 & 52 & 47 & 43 & 45 \\
\hline Juden & 2 & 3 & 4 & 21 & 25 & 19 & 78 & 74 & 79 \\
\hline Andere & 6 & 7 & 6 & 22 & 23 & 28 & 73 & 74 & 62 \\
\hline Keine & 12 & 10 & 9 & 23 & 31 & 30 & 75 & 67 & 61 \\
\hline Kirchgang & & & & & & & & & \\
\hline mehrmals/Woche & 12 & 16 & 14 & 55 & 64 & 63 & 43 & 35 & 36 \\
\hline Wöchentlich & 27 & 26 & 28 & 55 & 58 & 57 & 43 & 41 & 40 \\
\hline Monatlich & 15 & 14 & 14 & 46 & 50 & 46 & 53 & 49 & 51 \\
\hline Selten & 28 & 28 & 28 & 39 & 45 & 42 & 59 & 54 & 54 \\
\hline $\mathrm{Nie}$ & 16 & 15 & 14 & 30 & 36 & 32 & 67 & 62 & 61 \\
\hline
\end{tabular}




\begin{tabular}{|c|c|c|c|c|c|c|c|c|c|}
\hline \multirow[t]{2}{*}{ Wählergruppe } & \multicolumn{3}{|c|}{ Wähleranteil } & \multicolumn{3}{|c|}{ McCain/Bush } & \multicolumn{3}{|c|}{$\begin{array}{c}\text { Obama/Kerry/ } \\
\text { Gore }\end{array}$} \\
\hline & 08 & 04 & 00 & 08 & 04 & 00 & 08 & 04 & 00 \\
\hline $\begin{array}{l}\text { Parteiidentifikation } \\
\text { Demokrat }\end{array}$ & 39 & 37 & 39 & 10 & 11 & 11 & 89 & 89 & 86 \\
\hline Republikaner & 32 & 37 & 35 & 90 & 93 & 91 & 9 & 6 & 8 \\
\hline Unabhängiger & 29 & 26 & 27 & 44 & 48 & 47 & 52 & 49 & 45 \\
\hline Ideologie & & & & & & & & & \\
\hline Liberal & 22 & 21 & 20 & 10 & 13 & 13 & 89 & 85 & 80 \\
\hline Moderat & 44 & 45 & 50 & 39 & 45 & 44 & 60 & 54 & 52 \\
\hline Konservativ & 34 & 34 & 29 & 78 & 84 & 81 & 20 & 15 & 17 \\
\hline Wohnort & & & & & & & & & \\
\hline $\begin{array}{l}>500.000 \text { Einwohner } \\
50.000-500.000\end{array}$ & 30 & 13 & 9 & 35 & 39 & 26 & 63 & 60 & 71 \\
\hline Einwohner & 30 & 19 & 20 & 35 & 49 & 40 & 63 & 49 & 57 \\
\hline Vorstädte & 49 & 45 & 43 & 48 & 52 & 49 & 50 & 47 & 47 \\
\hline $10.000-50.000$ & & & & & & & & & \\
\hline Einwohner & - & 8 & 5 & - & 50 & 59 & - & 48 & 38 \\
\hline Ländliche Gegend & 21 & 16 & 23 & 53 & 59 & 59 & 45 & 40 & 37 \\
\hline Wahlentscheidung & & & & & & & & & \\
\hline heute & 4 & 5 & 11 & 45 & 45 & 46 & 50 & 52 & 48 \\
\hline Letzte drei Tage & 3 & 4 & 11 & 52 & 42 & 46 & 47 & 55 & 48 \\
\hline Letzte Woche & 3 & 2 & 6 & 50 & 51 & 44 & 48 & 48 & 48 \\
\hline Letzten Monat & 15 & 10 & 13 & 43 & 44 & 45 & 54 & 54 & 49 \\
\hline Früher & 74 & 78 & 69 & 47 & 53 & 50 & 52 & 46 & 48 \\
\hline
\end{tabular}

Prozentwerte. ${ }^{\text {a }}$ Die Daten für 2008 umfassen die Gruppe der 45-64-Jährigen, sind also mit den Vorjahren nicht vergleichbar. Quellen: CNN 2000, 2004, 2008.

Bei den ethnischen Gruppierungen war ebenfalls weitgehend Kontinuität zu beobachten: Weiße präferierten mehrheitlich den Republikaner McCain, Schwarze wählten fast ausschließlich den Demokraten Obama, und auch die amerikanischen Staatsbürger asiatischer Herkunft bevorzugten eindeutig den demokratischen Kandidaten. Eine Abweichung - oder präziser: eine Rückkehr zum historischen Trend war nur bei einer ethnischen Gruppierung, den Latinos, auszumachen. Allerdings sind hier die Befunde nicht über alle Zweifel erhaben: Die Stimmen der Latinos gewann Obama in einem Ausmaß, wie es 
nach dem guten Abschneiden Bushs bei dieser Wählergruppe in der Präsidentschaftswahl 2004 eigentlich nicht erwartet werden konnte. ${ }^{2}$ Auch hatte in der demokratischen Vorwahl Hillary Clinton diese Wählergruppe gegenüber Obama im Verhältnis von zwei zu eins gewonnen (Minushkin/Lopez 2008: 1). Da John McCain sich in der Einwanderungspolitik auf dem liberalen Flügel seiner Partei positioniert hatte, stand aus Sicht der Demokraten zu befürchten, dass die Latinos auch 2008 wieder in großer Zahl für den republikanischen Kandidaten votieren könnten (Greenblatt 2008: 461; Katel 2008: 590 f.). Diese Befürchtungen bewahrheiteten sich jedoch nicht; zwei Drittel aller Latinos wählte schließlich Obama. Damit lag Obama nach den beiden BushWahlen nahezu exakt wieder im Durchschnitt des demokratischen Latino Vote der Jahre 1976 bis 1996 (Leal u. a. 2005: 41). In Nevada, in New Mexico und auch in Florida mit seinem hohen Anteil an Einwanderern aus Kuba, die traditionell eher den Republikanern zuneigen, war die Unterstützung der Latinos für den Sieg Obamas sogar unverzichtbar (Lopez 2008; Tabelle 4).

Der im Vorfeld der Wahl viel diskutierte Bradley-Effekt, also der gegenüber geäußerten Wahlabsichtsumfragen möglicherweise zu erwartende Wählerumschwung der Weißen am Wahltage zugunsten von McCain, lässt sich den aggregierten Daten der Wahltagsumfrage nicht entnehmen. Dennoch hat es ihn im Einzelfall und auch regional begrenzt durchaus gegeben: In vier Staaten des tieferen Südens - in Alabama, Mississippi, Arkansas und Louisiana - wanderten die Weißen gegenüber 2004 noch einmal deutlich verstärkt von den Demokraten zu den Republikanern ab (Bartels, in: Brookings IV 2008: 14). Insgesamt wurde diese Wählerwanderung aber kompensiert durch die Zugewinne Obamas bei der weißen Bevölkerung

2 Die 44 \% für Bush in der Präsidentschaftswahl 2004, die sowohl der Voter Exit Poll von Edison/Mitofsky für den National Election Pool als auch der Exit Poll der Los Angeles Times auswiesen, sind in der Wissenschaft nicht unbestritten geblieben. Ein methodologisch anspruchsvollerer Exit Poll (jedoch mit kleinerer Stichprobe) des „William C. Velasquez Institute“, eines gemeinnützigen Forschungsinstituts, das sich die Verbesserung der politischen Partizipation von Latinos zum Ziel gesetzt hat, kam zu stark abweichenden Ergebnissen, die besser mit den Ergebnissen von Wahlabsichtsumfragen vor der Wahl harmonierten. Danach erzielte Bush 2004 nur 33,0 \% des Latino Vote, 65,4 \% wählten Kerry (Leal u. a. 2005; Flores 2004). Auf der Basis der Zensusdaten erscheint es begründet, die Zahl für Bush im Nachhinein auf etwa 40 \% zu korrigieren (Suro/Fry/Passel 2005: 11 ff.). Damit hätte Bush zwar immer noch das seit Jahrzehnten beste Ergebnis der Republikaner bei den Latinos erzielt, läge aber nicht mehr so unwahrscheinlich hoch über dem bisherigen Rekord von Ronald Reagan aus dem Jahre 1984 (37\%). 
anderswo, vor allem in Staaten des oberen Südens wie Virginia und den beiden Carolinas (Tabelle 5).

Tabelle 4

Das Latino Vote nach Einzelstaaten 2008 und 2004

\begin{tabular}{|l|c|c|c|c|c|c|}
\hline Einzelstaat & $\begin{array}{c}\text { Wähleran- } \\
\text { teil 2008 }\end{array}$ & Obama & McCain & $\begin{array}{c}\text { Wähleran- } \\
\text { teil 2004 }\end{array}$ & Kerry & Bush \\
\hline New Jersey & 9 & 78 & 21 & 10 & 56 & 43 \\
\hline Nevada & 15 & 76 & 22 & 10 & 60 & 39 \\
\hline California & 18 & 74 & 23 & 21 & 63 & 32 \\
\hline Illinois & 7 & 72 & 27 & 8 & 76 & 23 \\
\hline New Mexico & 41 & 69 & 30 & 32 & 56 & 44 \\
\hline Texas & 20 & 63 & 35 & 20 & 50 & 49 \\
\hline Colorado & 13 & 61 & 38 & 8 & 68 & 30 \\
\hline Florida & 14 & 57 & 42 & 15 & 44 & 56 \\
\hline Arizona & 16 & 56 & 41 & 12 & 56 & 43 \\
\hline USA & 9 & 67 & 31 & 8 & 53 & 44 \\
\hline
\end{tabular}

Prozentwerte; Quellen: CNN 2004, 2008; Lopez 2008.

Unentbehrlich für den Sieg Obamas war seine überproportionale Unterstützung durch die jüngeren Wähler: Die stärksten Zuwächse verzeichnete er bei den Erstwählern (plus 16 Prozentpunkte gegenüber 2004), bei den 18- bis 29-Jährigen $(+12)$ und auch noch bei den 30- bis 44-Jährigen $(+6)$. Bei den über 45 Jahre alten Wählern lag McCain dagegen gleichauf, bei den Wählern im Rentenalter lag er sogar vorne (Tabelle 3).

$\mathrm{Zu}$ weiteren besonders auffälligen - das soll heißen: überproportionalen oder unerwarteten - Zuwächsen zählten die folgenden: Bei den Wählern mit Kindern unter 18 Jahren im Haushalt, immerhin $40 \%$ der gesamten Wählerschaft, legte Obama acht Prozentpunkte zu und kehrte das Ergebnis von 2004 um. Hatte vor vier Jahren noch Bush bei dieser Gruppe mit 53 zu 45 \% vorne gelegen, so tat dies nun Obama mit identischem Vorsprung. Obama fand auch enormen Zuspruch bei den Ärmsten der Armen (Familieneinkommen unter 15.000 US-Dollar) und den Superreichen (über 200.000 US-Dollar). In der letzten Gruppe legte er gegenüber 200417 Prozentpunkte zu und lief seinem republikanischen Gegner den Rang ab. Beide Einkommensgruppen machten zusammen zwar nur $12 \%$ der Wählerschaft aus; dennoch legte dieser 
Befund Zeugnis ab über die besondere Fähigkeit Obamas, disparate Wählerkoalitionen zu schmieden. Eine ähnliche Spreizung im Wahlverhalten zeigten die nach Bildung unterschiedenen Wähler: Auch hier fand Obama den größten Rückhalt bei den formal am niedrigsten und den formal am höchsten gebildeten Wählern, während die mittleren Bildungsgruppen sich relativ gleichmäBig auf beide Kandidaten verteilten. Wenn Obama denn in irgendeiner Hinsicht als ein Kandidat der Extreme beschrieben werden kann, dann mit Blick auf die Einkommens- und Bildungsklassen seiner Anhängerschaft (Tabelle 3).

Tabelle 5

Wahlverhalten der Schwarzen und der Weißen in den Südstaaten 2008

\begin{tabular}{|l|c|c|c|c|c|c|}
\hline Einzelstaat & $\begin{array}{c}\text { Anteil } \\
\text { Schwarze }\end{array}$ & Obama & McCain & $\begin{array}{c}\text { Anteil } \\
\text { Weiße }\end{array}$ & Obama & McCain \\
\hline Virginia & $20(-1)$ & $92(+5)$ & $8(-4)$ & $70(-2)$ & $39(+7)$ & $60(-8)$ \\
\hline North Carolina & $23(-3)$ & $95(+10)$ & $5(-9)$ & $72(+1)$ & $35(+8)$ & $64(-9)$ \\
\hline Florida & $13(+1)$ & $96(+10)$ & $4(-9)$ & $71(+1)$ & $42(-)$ & $56(-1)$ \\
\hline & & & & & & \\
\hline South Carolina & $25(-5)$ & $96(+11)$ & $4(-11)$ & $71(+4)$ & $26(+4)$ & $73(-5)$ \\
\hline Georgia & $30(+5)$ & $98(+10)$ & $2(-10)$ & $65(-5)$ & $23(-)$ & $76(-)$ \\
\hline Alabama & $29(+4)$ & $98(+7)$ & $2(-4)$ & $65(-8)$ & $10(-9)$ & $88(+8)$ \\
\hline Kentucky & $11(+3)$ & $90(+3)$ & $8(-4)$ & $85(-5)$ & $36(+1)$ & $63(-1)$ \\
\hline Tennessee & $12(-1)$ & $94(+3)$ & $6(-3)$ & $84(-)$ & $34(-)$ & $63(-2)$ \\
\hline Mississippi & $33(-1)$ & $98(+8)$ & $2(-8)$ & $62(-3)$ & $11(-3)$ & $88(+3)$ \\
\hline Arkansas & $12(-3)$ & $95(+1)$ & $5(-1)$ & $83(-)$ & $30(-6)$ & $68(+5)$ \\
\hline Louisiana & $29(+2)$ & $94(+4)$ & $4(-5)$ & $65(-5)$ & $14(-10)$ & $84(+9)$ \\
\hline Oklahoma & $7(-2)$ & - & - & $82(+5)$ & $29(-)$ & $71(-)$ \\
\hline Texas & $13(+1)$ & $98(+15)$ & $2(-15)$ & $63(-3)$ & $26(+1)$ & $73(-1)$ \\
\hline
\end{tabular}

Prozentwerte; in Klammern Veränderungen gegenüber 2004 in Prozentpunkten. Quelle: CNN 2004, 2008.

Wie sehr Obama jedoch in Wirklichkeit ein Kandidat des Mainstream war, zeigte sich nicht zuletzt am Wahlverhalten der Konfessionsgruppen. Zunächst einmal ist auch hier vor allem Kontinuität festzustellen: Protestanten, vor allem weiße Protestanten und noch mehr weiße wiedergeborene Evangelikale, wählten überproportional republikanisch; Juden, Mitglieder anderer Religionsgemeinschaften und Konfessionslose wählten $\mathrm{zu}$ drei Vierteln 
demokratisch. Bemerkenswert ist jedoch, dass Obama, dessen Zugehörigkeit zur schwarzen Gemeinde einer evangelisch-reformierten Kirche (Trinity United Church of Christ) im Wahlkampf für negative Schlagzeilen gesorgt hatte (Rüb 2008), auch bei den weißen Protestanten leicht zulegen konnte. Für ein Viertel der weißen wiedergeborenen Christen, die zuverlässigste Klientel der Republikaner, erwies sich Obama als wählbar. Er gewann auch knapp die Hälfte der weißen katholischen Wähler für sich und legte dabei gegenüber 2004, als der Katholik John F. Kerry das demokratische ticket angeführt hatte, noch leicht zu. Dass Obama unter den Katholiken insgesamt die Mehrheit für die Demokraten zurückerobern konnte, hatte er aber vor allem den Latinos zu verdanken (Tabelle 3). Die Zeiten, in denen die Katholiken automatisch der demokratischen Wählerkoalition zugeschrieben werden konnten oder gar wie noch 1960 zu 83 \% für den demokratischen Kandidaten John F. Kennedy stimmten, sind unwiderruflich vorbei (Liedhegener 2005).

\section{Die Motive der Wahlentscheidung: Themen und Kandidaten}

Die bisher untersuchten geografischen und soziologischen Muster im Wahlverhalten sind notwendige Bestandteile einer umfassenden Erklärung des Wahlerfolgs von Barack Obama; sie reichen aber nicht aus, um die spezifischen Motive der Wahlentscheidung zu erfassen. Die Präsidentschaftswahl des Jahres 2008 stand ganz eindeutig im Zeichen der achtjährigen Präsidentschaft George W. Bushs und war - wie im Grunde auch schon die midterm elections des Jahres 2006 (Jacobson 2007b) - ein Referendum über dessen beide Amtszeiten. Kein Präsident der Nachkriegszeit mit Ausnahme von Harry S. Truman war am Ende seiner zweiten Amtszeit so unpopulär wie George W. Bush. Seine Zustimmungsrate von $25 \%$ am Vorabend der Präsidentschaftswahl 2008 wurde seit 1937, als Gallup diese Frage erstmals stellte, nur von Truman und Richard Nixon unterboten. ${ }^{3}$ Allerdings zogen selbst diese beiden bisher unpopulärsten amerikanischen Präsidenten nie eine solch hohe Ablehnung ihrer Amtsführung auf sich, wie sie Gallup im Oktober 2008 mit 71 \% für Bush ermittelte (Gallup 2008b; Roper Center 2008b). Die Wahltagsumfrage bestätigte indirekt die Bedeutung, welche die Unpopularität des amtierenden Präsidenten für den Ausgang dieser Wahl hatte: Von den

3 Andere Meinungsumfragen (z. B. für CBS/New York Times, ABC/Washington Post oder CNN/Opinion Research) ermittelten noch niedrigere Werte von minimal $22 \%$ für Bush. Für den historischen Vergleich eignen sich jedoch nur die Gallup-Daten, weil allein sie vollständig seit Franklin Delano Roosevelts Zeiten vorliegen. 
$27 \%$ der Wähler, die am 4. November ihre Zustimmung zur Amtsführung von Präsident Bush bekundeten, wählten 89 \% McCain, nur $10 \%$ Obama. Dagegen entschieden sich $67 \%$ derjenigen, die seine Amtsführung ablehnten - und das waren $71 \%$ aller Wähler - für Obama, nur $31 \%$ für McCain (Tabelle 6).

Die Daten der Wahltagsumfrage zeigen aber noch sehr viel unmittelbarer, dass die Wahl Obamas zuvörderst eine Abwahl Bushs war - und zwar in erster Linie aufgrund von dessen negativ bewerteter wirtschaftspolitischer Leistungsbilanz, erst in zweiter Linie aufgrund seiner umstrittenen Irakkriegspolitik. Für 63 \% der Wähler war die Wirtschaft das wichtigste Thema bei ihrer Wahlentscheidung, ein extrem hoher Wert im Vergleich zu früheren Wahlen (2004: 20, 2000: 18 und 1996: 21 \%). Selbst 1992, als Bill Clinton mit dem Slogan „It's the economy, stupid!“ Wahlkampf machte, hatte die Wirtschaft in der Wahrnehmung der Wähler nicht eine solche, alles andere überragende Bedeutung wie 2008 (Los Angeles Times 1992: 17). ${ }^{4}$ Dies bezeugen auch die selten pessimistischen Einschätzungen der ökonomischen Situation: Dass die Wirtschaftslage der Nation gut sei, meinten nur sieben Prozent der Wähler, $93 \%$ bewerteten sie als schlecht. Drei von vier Wählern sahen das Land darüber hinaus auf dem Weg in die falsche Richtung. Und ebenfalls drei Viertel aller Wähler sah ihre finanzielle Situation gegenüber 2004 als unverändert oder schlechter an. Ein eindeutigeres „Misstrauensvotum“ gegen einen amtierenden Präsidenten dürfte sich aus einer Wahltagsbefragung schwerlich ablesen lassen. (Tabelle 6).

4 Die Vergleichbarkeit ist nur annäherungsweise gegeben; der hohe Wert für 2008 wurde in gewisser Weise auch methodologisch erzeugt. Die Exit Polls wurden von verschiedenen Instituten (1992: Voter Research \& Surveys, 1996/2000: Voter News Service, 2004/2008: Edison Media Research/Mitofsky International) durchgeführt, die die Frage nach dem wichtigsten Thema (bzw. den wichtigsten Themen) immer etwas anders stellten. Zwar verwendeten alle Umfragen geschlossene Fragen, die Zahl der vorgegebenen Antworten (1992/96: 9, 2000/04: 7, 2008: 5) und der ankreuzbaren Felder (1992/96: ein oder zwei, 2000/04/08: ein) war jedoch unterschiedlich. Alle Fragebögen der Exit Polls seit 1972 dokumentiert Roper Center 2008a. 
Tabelle 6

Motive für die Wahlentscheidung bei der Präsidentschaftswahl 2008 laut Voter Exit Polls

\begin{tabular}{|c|c|c|c|}
\hline Motive & $\begin{array}{c}\text { Wähler- } \\
\text { anteil }\end{array}$ & Obama & McCain \\
\hline $\begin{array}{l}\text { Wichtigstes Thema } \\
\text { Wirtschaft } \\
\text { Irak } \\
\text { Terrorismus } \\
\text { Gesundheitsversorgung } \\
\text { Energiepolitik }\end{array}$ & $\begin{array}{r}63 \\
10 \\
9 \\
9 \\
7\end{array}$ & $\begin{array}{l}53 \\
59 \\
13 \\
73 \\
50\end{array}$ & $\begin{array}{l}44 \\
39 \\
86 \\
26 \\
46\end{array}$ \\
\hline $\begin{array}{l}\text { Amtsführung des Präsidenten } \\
\text { Zustimmung } \\
\text { Ablehnung }\end{array}$ & $\begin{array}{l}27 \\
71 \\
\end{array}$ & $\begin{array}{l}10 \\
67 \\
\end{array}$ & $\begin{array}{l}89 \\
31 \\
\end{array}$ \\
\hline $\begin{array}{l}\text { Entscheidung für den Irakkrieg } \\
\text { Zustimmung } \\
\text { Ablehnung }\end{array}$ & $\begin{array}{l}36 \\
63\end{array}$ & $\begin{array}{l}13 \\
76\end{array}$ & $\begin{array}{l}86 \\
22\end{array}$ \\
\hline $\begin{array}{l}\text { Wirtschaftslage der Nation } \\
\text { exzellent/gut } \\
\text { nicht so gut/schlecht }\end{array}$ & $\begin{array}{r}7 \\
93 \\
\end{array}$ & $\begin{array}{l}26 \\
54 \\
\end{array}$ & $\begin{array}{l}72 \\
44 \\
\end{array}$ \\
\hline $\begin{array}{l}\text { Land bewegt sich } \\
\text { in die richtige Richtung } \\
\text { falsche Richtung }\end{array}$ & $\begin{array}{l}21 \\
75\end{array}$ & $\begin{array}{l}28 \\
62 \\
\end{array}$ & $\begin{array}{l}71 \\
36\end{array}$ \\
\hline $\begin{array}{l}\text { Finanzlage der Familie } \\
\text { ist besser als vor } 4 \text { Jahren } \\
\text { schlechter } \\
\text { unverändert }\end{array}$ & $\begin{array}{l}24 \\
42 \\
34\end{array}$ & $\begin{array}{l}37 \\
70 \\
45\end{array}$ & $\begin{array}{l}60 \\
28 \\
53\end{array}$ \\
\hline $\begin{array}{l}\text { Ökonomische Lage wird im nächsten Jahr } \\
\text { besser } \\
\text { schlechter } \\
\text { bleibt wie sie ist }\end{array}$ & $\begin{array}{l}47 \\
23 \\
25\end{array}$ & $\begin{array}{l}61 \\
43 \\
52\end{array}$ & $\begin{array}{l}38 \\
54 \\
46\end{array}$ \\
\hline $\begin{array}{l}\text { Rassenbeziehungen werden künftig } \\
\text { besser } \\
\text { werden schlechter } \\
\text { bleiben wie sie sind }\end{array}$ & $\begin{array}{l}47 \\
15 \\
35\end{array}$ & $\begin{array}{l}70 \\
27 \\
44\end{array}$ & $\begin{array}{l}28 \\
70 \\
54\end{array}$ \\
\hline
\end{tabular}




\begin{tabular}{|c|c|c|c|}
\hline Motive & $\begin{array}{c}\text { Wähler- } \\
\text { anteil }\end{array}$ & Obama & McCain \\
\hline $\begin{array}{l}\text { Wichtigste Kandidatenqualität } \\
\text { kann Wechsel bringen } \\
\text { teilt meine Werte } \\
\text { hat Erfahrung } \\
\text { sorgt sich um die Menschen } \\
\end{array}$ & $\begin{array}{l}34 \\
30 \\
20 \\
12\end{array}$ & $\begin{array}{r}89 \\
31 \\
7 \\
73\end{array}$ & $\begin{array}{r}9 \\
66 \\
93 \\
24 \\
\end{array}$ \\
\hline $\begin{array}{l}\text { Obamas Positionen zu den Themen } \\
\text { zu liberal } \\
\text { zu konservativ } \\
\text { in etwa richtig }\end{array}$ & $\begin{array}{r}42 \\
4 \\
50\end{array}$ & $\begin{array}{r}9 \\
49 \\
93\end{array}$ & $\begin{array}{c}89 \\
44 \\
6\end{array}$ \\
\hline $\begin{array}{l}\text { McCain würde Bushs Politik } \\
\text { fortsetzen } \\
\text { nicht fortsetzen }\end{array}$ & $\begin{array}{l}48 \\
48 \\
\end{array}$ & $\begin{array}{l}90 \\
13 \\
\end{array}$ & $\begin{array}{r}8 \\
85 \\
\end{array}$ \\
\hline $\begin{array}{l}\text { Richtige Urteilskraft } \\
\text { nur Obama } \\
\text { nur McCain } \\
\text { beide }\end{array}$ & $\begin{array}{l}43 \\
36 \\
13\end{array}$ & $\begin{array}{r}98 \\
2 \\
64\end{array}$ & $\begin{array}{r}2 \\
97 \\
34\end{array}$ \\
\hline $\begin{array}{l}\text { Richtige Erfahrung } \\
\text { nur Obama } \\
\text { nur McCain } \\
\text { beide }\end{array}$ & $\begin{array}{l}32 \\
40 \\
19\end{array}$ & $\begin{array}{r}98 \\
3 \\
85 \\
\end{array}$ & $\begin{array}{r}1 \\
96 \\
13 \\
\end{array}$ \\
\hline $\begin{array}{l}\text { Rasse der Kandidaten } \\
\text { wichtigster Faktor } \\
\text { wichtiger Faktor } \\
\text { geringfügiger Faktor } \\
\text { kein Faktor }\end{array}$ & $\begin{array}{r}2 \\
7 \\
10 \\
80\end{array}$ & $\begin{array}{l}57 \\
51 \\
53 \\
51\end{array}$ & $\begin{array}{l}41 \\
48 \\
45 \\
46\end{array}$ \\
\hline $\begin{array}{l}\text { Alter der Kandidaten } \\
\text { wichtigster Faktor } \\
\text { wichtiger Faktor } \\
\text { geringfügiger Faktor } \\
\text { kein Faktor }\end{array}$ & $\begin{array}{r}2 \\
13 \\
23 \\
60\end{array}$ & $\begin{array}{l}74 \\
77 \\
59 \\
42\end{array}$ & $\begin{array}{l}26 \\
21 \\
39 \\
55\end{array}$ \\
\hline $\begin{array}{l}\text { McCains Wahl von Sarah Palin } \\
\text { wichtigster Faktor } \\
\text { wichtiger Faktor } \\
\text { geringfügiger Faktor } \\
\text { kein Faktor }\end{array}$ & $\begin{array}{r}7 \\
33 \\
20 \\
33\end{array}$ & $\begin{array}{l}52 \\
47 \\
33 \\
64\end{array}$ & $\begin{array}{l}47 \\
53 \\
66 \\
33\end{array}$ \\
\hline
\end{tabular}




\begin{tabular}{|l|c|c|c|}
\hline Motive & $\begin{array}{c}\text { Wähler- } \\
\text { anteil }\end{array}$ & Obama & McCain \\
\hline Nicht qualifiziert für das Präsidentenamt & 60 & 81 & 16 \\
Palin & 32 & 17 & 80 \\
Biden & 58 & 60 & 38 \\
\hline Themen oder Kandidaten & 39 & 39 & 59 \\
Themen wichtiger als Kandidaten & & & \\
Persönliche Qualitäten der Kandidaten & & & \\
wichtiger & 13 & 80 & 19 \\
Persönlich kontaktiert & 6 & 17 & 82 \\
nur von Obamas Wahlkampfstab & 13 & 47 & 51 \\
nur von McCains Wahlkampfstab & 66 & 50 & 48 \\
von beiden & & & \\
keinen Kontakt & 52 & 84 & 15 \\
\hline Bei Wahl zwischen Clinton und McCain & 41 & 7 & 91 \\
Clinton & 5 & 78 & 10 \\
McCain & &
\end{tabular}

Prozentwerte. Quelle: CNN 2008.

In wissenschaftstheoretischer Hinsicht kann das Wahlergebnis $2008 \mathrm{zu}-$ mindest teilweise als Rehabilitierung der Theorie des retrospektiven Wählens gedeutet werden (Key 1966; Fiorina 1981): Die Anhänger dieser Theorie waren nach dem Ausgang der Präsidentschaftswahl 2000, als die zentralen wirtschaftlichen Daten und Präsident Clintons hohe Zustimmungsraten am Ende seiner zweiten Amtszeit eigentlich einen deutlichen Sieg des demokratischen Kandidaten Al Gore über George W. Bush erwarten ließen, ins Zweifeln geraten. Alle sozialwissenschaftlichen Prognostiker in den USA, die sich professionell mit der Voraussage der Präsidentschaftswahlergebnisse befassen und dabei Erklärungsmodelle des retrospektiven Wählens bzw. des economic voting anwenden, hatten einen hohen Sieg Gores mit einem durchschnittlichen Stimmenanteil von $56 \%$ des two-party vote prophezeit und damit sein tatsächliches Ergebnis um knapp sechs Prozentpunkte überschätzt (Wlezien 2001: 27). Erklärungen für ihre „Über-Performanz“ fanden die meisten Prognostiker nach der Wahl schnell in der „Unter-Performanz" Gores: Als Vizepräsident konnte er den Amtsinhaberbonus nicht für sich reklamieren, wollte es augenscheinlich auch gar nicht, wie die Distanzierung von 
Clinton im Wahlkampf und seine Entscheidung, keinen retrospektiven, sondern einen prospektiven, linkspopulistischen Wahlkampf zu führen, gezeigt hatten. Auch mochte der im dritten Quartal 2000, nach Abgabe der Prognosen, einsetzende wirtschaftliche Abschwung das Übrige dazugetan haben, dass Gore hinter den Erwartungen zurückgeblieben war (Campbell 2001; Fiorina/Abrams/Pope 2003).

2008 war dagegen - wie auch schon 2004 - ein besseres Jahr für die in der American Political Science Association aktiven professionellen Prognostiker: Sieben der zehn Prognosen sagten einen Sieg Obamas voraus, eine sah McCain vorne und zwei prophezeiten ein Kopf-an-Kopf-Rennen. Die durchschnittliche Vorhersage für McCain von $48,0 \%$ des two-party vote lag nur 1,7 Prozentpunkte über dem tatsächlichen Ergebnis und stellte damit sogar eine nochmalige Verbesserung gegenüber 2004 dar. Damals hatten zwar sechs von sieben Prognostikern den Sieg Bushs vorausgesehen und nur ein Forscherduo ein Kopf-an-Kopf-Rennen prophezeit. Das schließlich erreichte Ergebnis Bushs hatten sie jedoch noch um durchschnittlich 2,6 Prozentpunkte überschätzt (Berechnungen nach Campbell 2004: 734; 2008: 681). Die guten Ergebnisse der „Prognoseindustrie“ im Jahre 2008 sollten nun aber auf der anderen Seite nicht dazu führen, die Erklärungskraft der Modelle retrospektiven Wählens zu überschätzen. 2008 war ein selten anzutreffender Idealfall für dieses Erklärungsmodell, weil sowohl die Ablehnung des amtierenden Präsidenten und seiner Partei wie auch das Ausmaß der Wirtschaftskrise Rekordwerte erreichten. Die Abstrafung einer regierenden Partei, die wirtschaftspolitisch versagt hat, fällt offensichtlich leichter als die Belohnung einer Partei im wirtschaftlichen Erfolgsfall.

Außerdem fließen beim economic voting nicht nur retrospektive Urteile über die Leistungsbilanz der regierenden Partei, sondern auch zukunftsgerichtete Urteile über die erwartete Politik der Kandidaten und über deren Eigenschaftsprofil in die Wahlentscheidung mit ein: So votierten diejenigen Wähler, die ihre Entscheidung 2008 vor allem am Wirtschaftsthema ausrichteten, in der Größenordnung des Gesamtergebnisses für Obama. Auch bei den anderen wichtigen Themen in diesem Wahlkampf - der Irak-, der Gesundheitsund der Energiepolitik - sah sich die Mehrheit der Wähler besser durch Obama vertreten. Nur die neun Prozent der Wähler, für die Terrorismusbekämpfung die oberste Priorität darstellte, votierten ganz überwiegend für McCain. Insgesamt bewertete die Mehrheit der Wähler Obamas Positionen zu dem Themen als in etwa richtig (50\%) oder sogar zu konservativ (4\%), nur $42 \%$ sahen ihn als zu liberal an. Die Hälfte der Wähler war darüber hinaus der 
Auffassung, dass McCain - der immerhin einmal eine Reputation als maverick in der Republikanischen Partei genossen hatte - die Politik Bushs im Wesentlichen fortführen würde und folgte damit einem zentralen Wahlkampfargument Obamas (Tabelle 6).

Die Wähler bewerteten auch das Eigenschaftsprofil Obamas günstiger als das seines Kontrahenten: So lag Obama in der Bewertung der Urteilskraft vor McCain, der seinerseits nur mit seiner Erfahrung gegenüber Obama punkten konnte. Diese erwies sich jedoch als ein zweischneidiges Schwert, thematisierte sie doch zugleich das Alter und den Gesundheitszustand des schon einmal an Krebs erkrankten Senators aus Arizona. Von den insgesamt knapp 40 \% der Wähler, für die das Alter der Kandidaten in irgendeiner Weise eine Rolle bei ihrer Wahlentscheidung spielte, entschied sich eine große Mehrheit für Obama. Dessen ,problematischste" Eigenschaft, seine Hautfarbe, schadete ihm dagegen nicht: Von den nur $20 \%$ der Wähler, für die Obamas Hautfarbe überhaupt eine Rolle spielte, entschied sich ebenfalls die Mehrheit für ihn. Insgesamt verkörperte der Kandidat Obama in geradezu idealtypischer Weise das Profil des Hoffnungsträgers: Ein Drittel der Wähler traute ihm zu, den politisch gewünschten Richtungswechsel vollbringen zu können. Knapp die Hälfte der Wahler, überwiegend Anhänger Obamas, erwartete angesichts der schweren Wirtschaftskrise Ende 2008 fast schon Wunderdinge vom künftigen Präsidenten und zeigte sich zuversichtlich, dass die ökonomische Situation im nächsten Jahr besser werde (Tabelle 6).

Abschließend ist noch einmal zu betonen, dass die Kandidatenbeurteilungen bei dieser Wahl nur von nachrangiger Bedeutung waren und eindeutig hinter den Themen zurückstanden. Die Themen ließen sich diesmal auf eines, maximal zwei reduzieren: Wirtschaft und Irak - und sie verdichteten sich beide zusammen in der negativen Bewertung von Präsident Bush, des seit über 50 Jahren unpopulärsten Präsidenten der USA. Unter den gegebenen Vorzeichen hätte wahrscheinlich jeder demokratische Präsidentschaftskandidat 2008 gewonnen, mit Sicherheit aber eine Präsidentschaftskandidatin Hillary Clinton. Laut Wahltagsumfrage hätte sie in etwa das gleiche Ergebnis wie Barack Obama erzielt, wahrscheinlich bei einer etwas niedrigeren Wahlbeteiligung (Tabelle 6).

\section{Der Beitrag der Wahlkämpfe zum Erfolg Obamas}

Nach den bisherigen Ausführungen könnte der Eindruck entstanden sein, dass sich weitere Erörterungen über die Bedeutung des Wahlkampfs für den 
Wahlerfolg Obamas erübrigen. Ganz falsch ist dieser Eindruck nicht, denn mit Sicherheit hat Obama die Präsidentschaftswahl 2008 nicht aufgrund seines besonders innovativen und fehlerfreien Wahlkampfs gewonnen (Nagourney/ Rutenberg/Zeleny 2008). Die nicht nur an Stammtischen, sondern auch akademisch diskutierte Frage, ob Wählkämpfe angesichts ihres kaum messbaren Einflusses nicht überflüssig seien - „Do Campaigns Matter?“ - geht dennoch an der Realität vorbei. So klein die Stimmenzugewinne durch effektive Wahlkämpfe auch sein mögen, kein Wahlkämpfer würde sich der Möglichkeit berauben, diese Stimmen auch zu ernten (Horst/Bretthauer 2001: 408). Darüber hinaus erfüllen Wahlkämpfe viele andere Funktionen für die Parteien, Kandidaten, Wähler und das politische System insgesamt, die sie schlichtweg unverzichtbar machen.

In ganz besonderem Maße gilt dies für das politische System der USA, in dem Wahlkämpfe für die Parteien auch die Funktion der internen Kandidatenauslese übernehmen. Wahlkämpfe sind hier mindestens ebenso sehr wie das alltägliche politische Handwerk in Legislative und Exekutive das Metier, in dem Politiker ihre Professionalität unter Beweis zu stellen haben. Vielleicht lässt sich die Bedeutung von Wahlkämpfen in den USA pointiert so zusammenfassen: Ambitionierte Politiker müssen sich als exzellente Wahlkämpfer beweisen, um überhaupt eine Existenzberechtigung zu haben. Gute Wahlkämpfe gewinnen zwar keine Wahlen (alleine schon deshalb, weil die anderen in der Regel ihr Geschäft genauso gut beherrschen), aber als mangelhaft wahrgenommene Wahlkämpfe können auch sicher geglaubte Siege noch verspielen - siehe Al Gore im Jahre 2000 oder Hillary Clinton in der demokratischen Vorwahl 2008 (Green 2008).

Die genaue Analyse der Wahlkämpfe rechtfertigt sich allein schon damit, dass der spätere Sieger alle Klippen im Wahlkampf, an denen er hätte scheitern können - und amerikanische Wahlkämpfe sind ein gnadenloser, fast zwei Jahre währender Ausscheidungswettlauf - im Gegensatz zu seinem Kontrahenten erfolgreich umschifft haben muss. Es ist deshalb eine faszinierende und lohnenswerte Aufgabe, die über Sieg oder Niederlage entscheidenden Ereignisse und Themen im Wahlkampf zu identifizieren sowie die Strategien der Wahlkämpfer zu ihrer Bewältigung zu analysieren.

\section{a) Der Wahlkampf McCains}

Alle Wahlverlierer teilen das Schicksal, dass sie in der Rückschau immer einen schlechten Wahlkampf gemacht haben. Der ausgebuffte Wahlkämpfer 
John McCain - erfolgreich in zwei Repräsentantenhaus-, vier Senatswahlkämpfen, in sieben Staaten bei der republikanischen Präsidentschaftskandidatur 2000 und bundesweit 2008 - war sich dieses politischen Naturgesetzes bewusst und beteiligte sich deshalb nach seiner Niederlage auch nicht an den üblichen Schuldzuweisungen (Bumiller 2008). ${ }^{5}$ Auch hier soll nicht der billige Eindruck erweckt werden, als hätte McCain, nur weil er verloren hat, einen schlechten Wahlkampf geführt (Zelizer 2008). Im Gegenteil: McCain zog alle Register des modernen politischen Marketings; er hatte (zeitweise) die besten political consultants und pollster auf republikanischer Seite zur Hand; er war in den unterschiedlichen Formaten der Fernsehduelle ein sachkundiger, schlagfertiger und humorvoller Debattant, der dem coolen, siegessicheren Obama im Publikumsurteil vor allem deshalb unterlag (Jeffrey M. Jones 2008), weil er zu aggressiv wirkte (aber die Möglichkeit, nicht zu attackieren, hatte der in den Wahlumfragen zurückliegende McCain nicht!); und er war insgesamt unter den gegebenen Umständen wahrscheinlich der beste vorstellbare republikanische Kandidat. Nach acht Jahren ,imperialer" und „polarisierter" Präsidentschaft unter George W. Bush ${ }^{6}$ hätte kein Kandidat der Republikaner einfach dort weitermachen können, wo George W. Bush aufgehört hatte. Der maverick John McCain, im Jahre 2000 noch erbitterter Gegner Bushs in der republikanischen Präsidentschaftskandidatur, konnte einen Neuanfang der Republikaner glaubhafter repräsentieren als jeder andere Kandidat.

McCains Eigenbrötlerimage brachte aber auch spezifische Probleme mit sich, die seinen Wahlkampf immer wieder gefährdeten. Schon in der invisible primary (Buell 1996, Cohen u. a. 2008b) lag McCain während des gesamten Jahres 2007 in den Meinungsumfragen hinter Rudy Giuliani, dem ehemaligen Bürgermeister von New York. Im Einwerben von Spenden waren Giuliani und der frühere Gouverneur von Massachusetts Mitt Romney, der Millionen

5 Bereits im Juli, also noch mitten im laufenden Wahlkampf, äußerte sich McCain in einem Interview wie folgt (zit. bei Bumiller 2008): „Every book I've read about a campaign is that the one that won, it was a perfect and beautifully run campaign with geniuses running it and incredible messaging, etcetera... And always the one that lost, ,Oh, completely screwed up, too much infighting, bad people, etcetera.' So if I win, I believe that historians will say, 'Way to go, he fine-tuned that campaign, and he got the right people in the right place and as the campaign grew, he gave them more responsibility.' If I lose, people will say, 'That campaign, always in disarray."' Dieses Zitat sei all jenen zum Nachdenken anempfohlen, die sich professionell mit der Analyse von Wahlkämpfen beschäftigen.

6 Horst 2004, 2005; Sinclair 2006; Edwards/King 2007. 
aus seinem Privatvermögen beisteuerte, ebenfalls erfolgreicher als der Senator aus Arizona (Tabelle 7). McCain musste aufgrund der nur spärlich fließenden Spenden im Sommer 2007 einen Großteil seines teuren Wahlkampfstabs entlassen (Balz/Kornblut 2007; Nagourney 2008a), was ihn in den Umfragen noch weiter zurückwarf. Auch hatte McCain von allen Kandidaten die leidenschaftlichsten Widersacher an der sozialkonservativen, fundamental-religiösen Parteibasis. Die religiöse Rechte erkor sich zeitweilig den Schauspieler und law-and-order-Politiker Fred Thompson sowie den früheren Baptistenpfarrer und Gouverneur von Arkansas Mike Huckabee zu ihren Favoriten.

Am Ende aber setzte sich McCain durch - nicht zuletzt weil seine Konkurrenten weitaus größere Mängel als er offenbarten: Thompson ließ jede Leidenschaft während seiner Kampagne vermissen; Giuliani verfolgte eine haarsträubend unprofessionelle Wahlkampfstrategie, indem er Iowa und New Hampshire ausließ; und Huckabee erzielte zwar nach seinem Auftaktsieg in Iowa noch einige aufsehenerregende Erfolge (vor allem im Süden), war aber insgesamt zu sehr der Kandidat politischer Randgruppen. Lediglich Mitt Romney erwies sich als ernstzunehmender Kandidat, überzeugte aber aufgrund seiner eher liberalen Politik als Gouverneur von Massachusetts nicht mit der Strategie, sich nun als der konservativste Kandidat im republikanischen Bewerberfeld zu positionieren. ${ }^{7}$

Den größten Fehler im Wahlkampf machte John McCain dann angeblich mit der Nominierung von Sarah Palin zur Vizepräsidentschaftskandidatin. Doch auch das lässt sich im Nachhinein leicht sagen; zunächst sahen viele pundits in dieser Entscheidung einen genialen medialen Überraschungscoup (Baker 2008): Mit Palin hatte niemand gerechnet, weil sie in Washington, D.C. kaum jemand kannte. Als Newcomerin schien sie Obama den Anspruch, Agent des Wandels zu sein, streitig machen zu können. Manche kalkulierten, dass Palin enttäuschte Anhängerinnen Hillary Clintons ins republikanische Lager herüberziehen könnte. Außerdem war sie entschieden konservativ, gegen Abtreibung und für den Krieg im Irak (in den ihr ältester Sohn alsbald ausrücken sollte), womit sie die konservative Parteibasis begeisterte. Der republikanische Parteikonvent Anfang September in St. Paul-Minneapolis im Bundesstaat Minnesota wurde denn auch mehr zur Feier Palins als McCains. Der post-convention bounce in den Wahlumfragen zugunsten des republikanischen tickets - McCain überflügelte für knapp zwei Wochen Obama im daily tracking poll (Gallup 2008a) - war eindeutig ihr Verdienst. Erst mit

7 Cohen u. a. 2008a; Mayer 2008; Walker 2008. 
der Zeit wurde immer deutlicher, dass McCain sich mit seiner Entscheidung „,verpokert“ hatte. Sie schlug ihm sein bestes, im Sommer ständig wiederholtes Argument, dass Obama nicht über die notwendige Erfahrung für das Präsidentenamt verfüge, aus der Hand. Auch warf sie ein zweifelhaftes Licht auf seine Urteilskraft und seinen Charakter, riskierte McCain es doch mit dieser Entscheidung, dass eine nicht ausreichend qualifizierte Kandidatin bei einem republikanischen Wahlsieg nur einen Herzschlag vom Präsidentenamt entfernt wäre. Ein solches Risiko aus wahlkampftaktischem Kalkül einzugehen stieß auch prominenten Republikanern unangenehm auf (Baker 2008; Zelizer 2008).

\section{Tabelle 7}

Einnahmen (Spenden und staatliche Zuschüsse) für Vorwahl- und Hauptwahlkampagne der Präsidentschaftskandidaten 2007/08, in Millionen USDollar

\begin{tabular}{|c|c|c|c|c|}
\hline & Obama & Clinton & McCain & Romney \\
\hline 1. Quartal 2007 & 25,8 & 36,1 & 14,8 & 23,4 \\
\hline 2. Quartal 2007 & 58,9 & 63,1 & 24,7 & 44,4 \\
\hline 3. Quartal 2007 & 80,3 & 90,9 & 32,1 & 62,8 \\
\hline 4. Quartal 2007 & 103,8 & 118,3 & 42,1 & 90,1 \\
\hline 31.01 .2008 & 140,6 & 138,0 & 54,8 & 106,8 \\
\hline 29.02 .2008 & 197,3 & 173,9 & 66,4 & 110,9 \\
\hline 31.03 .2008 & 240,2 & 194,8 & 81,9 & 111,0 \\
\hline 30.04 .2008 & 272,1 & 221,7 & 100,4 & 111,1 \\
\hline 31.05 .2008 & 295,5 & 238,0 & 121,9 & 113,5 \\
\hline 30.06 .2008 & 340,5 & 236,8 & 140,9 & 112,1 \\
\hline 31.07 .2008 & 390,9 & & 177,2 & \\
\hline 31.08 .2008 & 456,1 & & 216,4 & \\
\hline 30.09 .2008 & 607,0 & & & \\
\hline 15.10 .2008 & 643,1 & & & \\
\hline 24.11 .2008 & 741,7 & & $345,6^{\mathrm{a}}$ & \\
\hline
\end{tabular}

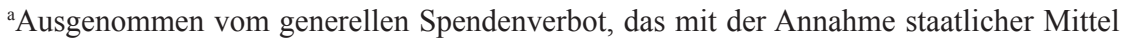
verbunden ist, sind Spenden zur Finanzierung von General Election Legal and Accounting Compliance Costs (GELAC). McCain hat seit Anfang September neben den staatlichen Zuschüssen von 84,1 Millionen noch 45,1 Millionen Dollar solcher GELAC-Spenden eingenommen. Quellen: CFI 2008; FEC 2008. 
Andere angebliche Fehler McCains - wie seine chaotische Wahlkampforganisation, sein ausgeprägtes negative campaigning, seine nicht vorhandene Electoral College-Strategie, sein Handling der Finanzkrise oder seine verspätete Positionierung in der Steuerpolitik (Zelizer 2008) - sollen hier nicht en détail beleuchtet werden, weil solche Kritik zum einen in der Nachbetrachtung wohlfeil und zum anderen nicht zu erkennen ist, dass diese „Fehler" wirklich zur Niederlage McCains entscheidend beigetragen haben (Bartels, in: Brookings IV 2008: 12). Zu berücksichtigen ist ferner, dass manche Entscheidungen McCains unter insgesamt günstigeren Vorzeichen für seinen Wahlkampf eine wohlwollendere Interpretation seitens der pundits gefunden hätten. Andere Entscheidungen hätte McCain gar nicht erst so getroffen, wie er sie dann traf, wenn er sich nicht bestimmten Zwangslagen der Unterfinanzierung seines Wahlkampfs, der Unbeliebtheit in sozialkonservativen Kreisen oder der Notwendigkeit, das positive Image Obamas anzugreifen - ausgesetzt gesehen hätte. Die Entscheidung für Palin zählte auch dazu: McCain wollte ursprünglich den demokratischen Senator von Connecticut Joe Lieberman nominieren, scheiterte damit aber in der Partei.

\section{b) Der Wahlkampf Obamas}

Obamas Wahlkampf ist einhellig - von demokratischer wie republikanischer, von journalistischer wie wissenschaftlicher Seite - gelobt worden (Brookings 2008; Nagourney/Rutenberg/Zeleny 2008), im Folgenden seien sieben der wichtigsten Punkte erörtert, einige weitere bloß angeführt:

Erstens: Der vielleicht erfolgreichste Schachzug der Obama-Kampagne bestand darin, McCain in die Tradition Bushs zu stellen. Dies lag angesichts der negativen Popularität des amtierenden Präsidenten nahe, doch auch Naheliegendes will entdeckt sein. Immer wieder hämmerte Obama den Slogan heraus, dass eine Wahl McCains gleichbedeutend sei mit einer dritten Amtszeit Bushs: „four more years Bush“. Am Wahltag identifizierte die Hälfte der Wähler McCain mit den politischen Positionen Bushs (Tabelle 6).

$\mathrm{Zu}$ dieser Identifizierung McCains mit Bush trug, zweitens, Obamas Fernsehwerbung bei, die professionell auf der Klaviatur der verschiedenen Formate spielte. Die Medienberater hatten in der Telegenität des Kandidaten, seiner Bilderbuchfamilie und seiner kraftvollen Rhetorik „Spielmaterial“" zur Verfügung, wie es idealer kaum hätte ausfallen können. Ein beeindruckender „Cinéma vérité"-Spot, der Obama zeigt, wie er ein großes Publikum in Bann zieht, war zum Beispiel der vor den Iowa-Caucuses gedrehte „Defining 
Moment"-Spot im 60-Sekunden-Format. Auch den „Sainthood“-Spot „Opportunity“ vom März 2008, in dem Obamas Leben erzählt wird, drehte das Team im längeren 60-Sekunden-Format. Im 30-Sekunden-Standardformat kamen die beiden „Cinéma vérité"-Spots „Commander in Chief“" und „President" daher, mit denen das Obama-Team im April 2008 auf McCains und Clintons Versuche reagierte, sich als diejenigen Kandidaten zu präsentieren, die über Erfahrung und Eignung für das Präsidentenamt verfügten. Clintons in diesem Zusammenhang gedrehter ,3 a.m."-Spot - die Anleihen beim berüchtigten „Daisy“-Spot von Lyndon B. Johnson aus dem Wahlkampf 1964 waren unübersehbar - war ein meisterhaftes Beispiel für intelligentes negative campaigning. Doch auch Obamas Medienprofis verstanden sich auf die Kunst der Negativwerbung - erkennbar am „Fundamentals“-Spot, der eine von McCain auf dem Höhepunkt der Finanzkrise gemachte Bemerkung aufgriff, wonach die Grundlagen der US-amerikanischen Wirtschaft in bestem Zustand seien. ${ }^{8} \mathrm{Zu}$ besonderer Meisterschaft brachten es die Wahlkampfstrategen Obamas im sofortigen Zurückschlagen von Angriffen; sie zogen damit die Lehren aus der Niederlage John F. Kerrys im Jahre 2004, der auf die Attacken der Swift Boat Veterans of Truth nicht reagiert hatte (Freedman 2006). Ein Beispiel für einen solchen counterattack- oder back-and-forth-ad war Obamas „Dime“-Spot, mit dem er innerhalb von 24 Stunden den „Pocket“Spot Hillary Clintons konterte, in dem sie ihm Interessenpolitik für große Ölunternehmen vorgeworfen hatte (New York Times 2008).

Im Zusammenhang seiner teuren Fernsehkampagne erwies es sich, drittens, als vorteilhaft, dass Obama entgegen früherer Äußerungen auf die öffentliche Finanzierung in der Hauptwahlkampfphase verzichtete (für die Vorwahlkampfphase hatten sie alle aussichtsreichen Kandidaten ausgeschlagen). Er war damit der erste Präsidentschaftskandidat seit Inkrafttreten der öffentlichen Finanzierung 1976, der weder im Vorwahl- noch im Hauptwahlkampf einen Cent öffentlichen Geldes annahm (Corrado 2005, 2006). Obama konnte so die mit der staatlichen Finanzierung verbundenen Ausgabengrenzen umgehen und seinen Geldvorteil gegenüber McCain weiter ausbauen.

8 Der Spot ist eingestellt auf http://www.youtube.com/watch?v=6reQLzgywzk\&feature=user (Stand: 16.01.09). Auch der „Daisy“-Spot Johnsons ist bei youtube zu finden. Alle anderen hier erwähnten Spots sind einzusehen bei New York Times 2008. Dort auch weitere Beispiele für gut gemachte negative ads - wie den „Celebrity“-Spot des McCain-Teams oder den „100 years“-Spot des Democratic National Committee - sowie klassische man-in-the-street-, news look-, talking head- oder issue position-Spots des Obama-Teams. Die Typisierung der verschiedenen Werbespots folgt Polsby/ Wildavsky 2008: $182 \mathrm{f}$. 
Während McCain ab September 2008 mit den staatlichen Zuschüssen von 84,1 Millionen Dollar auskommen musste, durfte Obama in den letzten beiden Monaten soviel Geld ausgeben, wie er einwarb. Allein im September nahmen seine Spendensammler die Rekordsumme von 150 Millionen Dollar ein. Nachdem die Fundraising-Operation in der ersten Oktoberhälfte etwas ins Stocken geraten war, kam Obama in den letzten drei Wochen noch auf die gigantische Summe von 741,7 Millionen Dollar für den gesamten Wahlzyklus 2007/08. Zum Vergleich: Bush und Kerry hatten 2004 zusammen 674,3 Millionen Dollar eingesammelt (Corrado Handout, in: Brookings IV 2008). McCain hatte mit 345,6 Millionen Dollar - 261,5 Millionen selbst eingeworben, 84,1 Millionen aus der Staatskasse - nicht einmal die Hälfte der Mittel Obamas zur Verfügung (Tabelle 7). McCain musste deshalb versuchen, mit Mitteln der Partei (Republican National Committee) Obamas Geldvorteil zu kompensieren, was ihm nur begrenzt gelang und mit Einbußen an werberischer Qualität und message control verbunden war. Obama dagegen konnte es sich im Endspurt des Wahlkampfes leisten, ein halbstündiges, mithilfe von Oscar-Preisträger Davis Guggenheimer gedrehtes Infomercial auf insgesamt sieben Fernsehsendern (darunter die drei großen Fox, NBC, CBS und ein spanischsprachiger Kanal) zu senden. Insgesamt sahen am 29. Oktober 2008 über 33 Millionen Zuschauer Obamas Werbefilm - das parallel gesendete Endspiel der World Series im Baseball verfolgten nur knapp 20 Millionen (Carter 2008).

Das halbstündige Infomercial ist ein gutes Beispiel für die positive Aura, die, viertens, Obamas gesamte Werbekampagne umgab. Zwar verzichtete er keineswegs auf negative oder counterattack ads (z. B. gegen die Versuche der Republikaner und Clintons, ihn in die Nähe von William Ayers, im Jahre 1969 Gründer der linkterroristischen Gruppe Weather Underground, zu rücken), dennoch blieb insgesamt der Eindruck einer positiven Kampagne haften, weil er anders als seine Kontrahenten mit seinen Attacken nie persönlich wurde. Auch hatte er aufgrund seiner gut gefüllten Wahlkampfkasse viel größeren Spielraum, positive Werbespots zu drehen. Obamas Slogans „Change, you/we can believe in“, „Yes, you/we can“, „We're One Nation: Our Time for Change has come“, „Bringing Democrats and Republicans together“, „America: Our Moment is now!“- waren dabei perfekt aufeinander abgestimmt und kreisten alle um das Change-Motiv und den Amerikanischen Traum. Obama traf mit seiner patriotischen Kampagne Amerika mitten ins Herz - und er verkörperte auch als Person (mitsamt seiner Familie) den Anspruch, Amerikas Versprechen zu erneuern (DNCC 2008). Das Wechselmotiv 
erwies sich in dieser Wahl als unschlagbar: es kündigte nicht nur das Ende der Bush-Administration an („Ending War in Iraq“), sondern es schien auch den Generationenwechsel in Washington einzuläuten. Alle persönlichen Attacken McCains oder Clintons konnte Obama erfolgreich zurückschlagen, indem er sie mit dem Fluch der „Same Old Politics“ belegte (New York Times 2008).

Obama überwand auch, fünftens, die zeitweilig größte Gefahr für seinen Wahlkampf, die ihn in Gestalt des schwarzen Reverend Jeremiah A. Wright Jr. heimsuchte. Wright, Anhänger einer schwarzen Befreiungstheologie, hatte Obama 1992 zum Eintritt in seine Chicagoer Kirche bewogen, ihn getraut und seine beiden Töchter getauft. Im Wahlkampf tauchten immer wieder Videos auf, die Wright bei wütenden Predigten zeigten, in denen er Amerika für den Rassismus, die „Erfindung“ der AIDS-Krankheit und die Provokation der Terroranschläge vom 11. September 2001 verdammte. Im Vorfeld der Pennsylvania-Primary gewann dieses Thema eine solche - von den Republikanern und Clinton angefachte - Dynamik, dass sich Obama von den ,aufrührerischen und entsetzlichen“ Äußerungen seines Pastors distanzierte und eine vielgerühmte, große Rede über die Rassenfrage hielt, in der er eine vermittelnde Position einnahm.

Obama warb um historisches Verständnis für den Zorn Wrights, der hier auch stellvertretend für die ältere Generation der schwarzen Bürgerrechtler stand, hatte aber auch ein offenes Ohr für weiße Angehörige der Arbeiterschaft und Mittelschichten, die ihr Ärger über schwarze welfare queens, Gewaltkriminalität oder Affirmative Action in die Arme Reagans geführt hatte (Obama 2008a). Zugleich weigerte er sich zum damaligen Zeitpunkt noch, Wright zu verleugnen, rechnete ihn weiter zum Freundeskreis. Die Verbindungen zu Wright kappte Obama erst, nachdem dieser sich nach der - von Obama verlorenen - Vorwahl von Pennsylvania auf einen Werbefeldzug in eigener Sache machte und seine Äußerungen sogar noch verschärfte. Eine Woche später gewann Obama die Vorwahl in North Carolina überzeugend und verlor nur hauchdünn in Indiana. Die Waage der Superdelegierten neigte sich mit Macht seiner Seite zu, die demokratische primary war faktisch entschieden.

Die Episode Wright steht exemplarisch für die erfolgreiche Bewältigung des Rassenthemas durch Obama, der eben weder zu schwarz, noch zu weiß war, sondern in seiner Person - und mit Ehefrau Michelle an seiner Seite, die ihm besonders in der schwarzen community half (Brinkbäumer 2009: 80) das Ende traditioneller black politics ankündigte. Die größten Widerstände 
hatte Obama nicht bei weißen Rassisten, sondern unter älteren schwarzen Aktivisten aus der Bürgerrechtsbewegung zu überwinden (Bai 2008). Und dennoch würde auch ein Reverend Jesse Jackson Sr., Präsidentschaftskandidat der Jahre 1984/88 und wie Wright unbeugsamer Anwalt der Schwarzen, bei Obamas Siegrede in Chicago weinen - ein Knäuel aus Freude und Leid, aus Stolz und Scham auflösend.

Die Einstellung der älteren schwarzen, größtenteils in Bürgerrechtsbewegung und Kirche sozialisierten Politiker hatte noch aus einem weiteren Grund Bedeutung für den Wahlkampf Obamas - und das betraf, sechstens, dessen ausgefeilte Delegiertenstrategie für den demokratischen Parteikonvent im August 2008 in Denver, Colorado. Obamas Wahlkampfteam um Chefstratege David Axelrod war sich von vornherein bewusst, dass es diesmal auch zu einem Showdown auf dem Parteikonvent, zu einer brokered convention kommen könnte und es deshalb auf jede einzelne Delegiertenstimme ankam. Anders als Hillary Clinton setzte Obamas Team nicht allein auf die großen bevölkerungsreichen Staaten, die im Electoral College stark zu Buche schlagen, sondern umwarb alle Staaten, auch die kleinen, bis hinein in den letzten Wahlsprengel. Das waren oftmals auch die Staaten, die ihre Delegierten auf Parteiversammlungen (Caucuses) und nicht per Vorwahlen nominieren (Walker 2008). Hier kam Obama der Enthusiasmus seiner Anhänger und seine ausgreifende grassroots campaign zugute. In manchen Staaten, die Clinton nach Stimmen gewann, heimste er mehr Delegierte ein als sie, weil die Demokraten ihre Delegierten eben nicht nach dem Winner-Takes-AllPrinzip, sondern aufgrund ausgefeilter Proporzformeln zuweisen (Polsby/ Wildavsky 2008: 113-143; Cost 2008).

Obama lag nach seinem Auftaktsieg in Iowa in der Delegiertenzählung (pledged delegates) immer leicht vor seiner Konkurrentin, die anfangs jedoch einen komfortablen Vorsprung bei den superdelegates hatte. Unter diesen 796 Ex-Officio-Delegierten, die knapp $20 \%$ aller Konventsdelegierten ausmachten, befand sich eine beträchtliche Anzahl schwarzer Politiker, die zunächst Clinton unterstützt hatten - aus unterschiedlichen Motiven, oft aber auch, weil sie nicht an die Wählbarkeit eines Schwarzen in der Hauptwahl glaubten. Die Superdelegierten gerieten ab dem 5. Februar 2008, als Obama auch die Mehrzahl der Staaten am Super Tuesday gewann, hinsichtlich ihrer Entscheidung unter zunehmenden Druck. Obama erhöhte diesen Druck, indem er seine vom ehemaligen Senate Majority Leader Tom Daschle angeführten „Kopfjäger“" verstärkt um ihre Stimme buhlen ließ. Auch formulierte er den Anspruch, dass die superdelegates auf ihren Entscheidungsspielraum 
verzichten und den nach Delegiertenzahl führenden Kandidaten akklamieren sollten (Barnes 2008). In der Folge schmolz der Vorsprung Clintons bei den Superdelegierten dahin; nach Obamas Sieg in North Carolina Anfang Mai 2008 übernahm er auch in dieser inoffiziellen Wertung die Führung.

Die grassroots campaign, die Obama im Laufe seines Vorwahlkampfes gegen Hillary Clinton auf- und weiter ausgebaut hatte, sollte, siebtens, eine entscheidende Voraussetzung für seinen Sieg in der Hauptwahl gegen John McCain bilden. Es ist viel von der überlegenen Wahlkampforganisation Obamas die Rede gewesen, seinem beeindruckenden fundraising - sowohl bei den Großspendern als auch bei den Kleinspendern über das Internet. Ende Okober 2008, so wurde berichtet, hatte er über drei Millionen Spender in seiner Kartei (Corrado, in: Brookings IV 2008: 6 f.). Weil er das Internet darüber hinaus mithilfe interaktiver Systeme wie Facebook und MySpace zum social networking nutzte, konnte er auch seine Freiwilligen-Organisation auf ein ungeahnt breites Fundament stellen: 13 Millionen E-Mail-Adressen soll er über sein Webseite eingesammelt haben (Walker 2008).

Obama errichtete Wahlkampfbüros in Flecken, die seit Jahrzehnten von keiner campaign heimgesucht worden waren - und er baute solche Büros auch in „tiefroten“ republikanischen Landstrichen auf, in die sich seit Jahrzehnten kein Demokrat mehr gewagt hatte. Ein Forscher hat sich einmal die Mühe gemacht, die Zahl der Wahlkampfbüros Obamas im Vergleich zu denen McCains in sechs battleground states (Florida, Iowa, Pennsylvania, Indiana, North Carolina, New Mexico) auszuzählen: Obama hatte 345, McCain 177 (Corrado, in: Brookings IV 2008: 8 f.). Im Hauptwahlkampf bildete dieses flächendeckende Netz von Büros, überwiegend ausstaffiert mit begeisterungsfähigen Freiwilligen, die Basis für die expansive Electoral CollegeStrategie Obamas. Je günstiger sich die Wahlabsichtsumfragen ab Ende September 2008 für ihn entwickelten, desto mehr konnte er seine Wahlkampfressourcen in diejenigen battlegrounds lenken, die traditionell eher den Republikanern zuneigten: Virginia, North Carolina und Indiana oder im Wahlkampfendspurt Colorado, Missouri und Nevada (Shaw 2006; Nagourney 2008b).

Über diese sieben hier erörterten Punkte hinaus trugen noch viele andere Faktoren und Entscheidungen Obamas - etwa die Wahl des außenpolitisch erfahrenen Joe Biden zu seinem Vizepräsidentschaftskandidaten oder Obamas Coolness und Sachkompetenz in den TV-Duellen - zu seinem erfolgreichen Wahlkampf bei. Die Entscheidungen sind, aus der Rückschau betrachtet, gut ausgegangen und waren somit zumindest nicht falsch. Man sollte sich aber der Tatsache bewusst bleiben, dass alle Entscheidungen Obamas durch die 
günstigen Vorzeichen, unter denen der Wahlkampf eines demokratischen Kandidaten am Ende der Ära George W. Bush stand, erleichtert worden sind. Unter weniger günstigen Umständen hätte zum Beispiel die Wahl Bidens auch als ein Eingeständnis der eigenen außenpolitischen Unerfahrenheit und die Coolness in den TV-Duellen als Arroganz gewertet werden können. Bei alldem schadete es nicht - und war nach acht Jahren Bush auch verständlich -, dass die Medien und das Pressekorps in Washington dem kommenden Präsidenten fast schon so etwas wie Bewunderung entgegenbrachten (Leibovich 2008).

\section{Ausblick: Vom Wahlkampf zum Regieren}

Wie auch immer der Stellenwert des Wahlkampfes für den Wahlerfolg am Ende eingeschätzt wird, am Wahltag ist der Wahlkampf vorbei und es beginnt der Übergang zum Regieren, die elfwöchige transition bis zur Inauguration des Präsidenten am 20. Januar des Folgejahres (Charles O. Jones 1998, 2007: 69-108). Regieren und Wahlkampf sind unterschiedliche Dinge, und doch haben sie manches gemein, sind eng miteinander verwoben. Manche Politikwissenschaftler monieren den - allerdings kommunikationstechnologisch als beinahe unvermeidlich angesehenen - Trend zur permanent campaign (Ornstein/Mann 2000) oder zum governing by campaigning (Edwards 2007), weil er dem auf Kompromissbildung und Konsenssuche angelegten Regieren die konfrontative Logik der Kampagne, des „Wir gegen Sie“, aufzwinge. Eine der politikwissenschaftlich interessanteren Fragenkomplexe der Präsidentschaft Obamas wird sein, inwieweit der begnadete Wahlkämpfer Obama, Kopf der ultimativen „21 $1^{\text {st }}$ Century Campaign” (Brownstein 2008), den Übergang vom Wahlkampf zum Regieren bewältigt, inwieweit er Elemente seiner grass roots campaign ins Regierungsgeschäft hinüberretten und die konfrontative Logik des governing by campaigning tatsächlich durch einen stärker kooperativ ausgerichteten Regierungsstil des Verhandelns ersetzen kann.

Seine bisherigen Entscheidungen in der transition deuten immerhin an, dass er gerne einen kooperativen Weg gehen würde. Zwar hat er um die Jahreswende herum mit bundesweit mehr als 4.000 house partys experimentiert, auf denen die Teilnehmer ihre Vorschläge zur Gesundheitsreform diskutierten, und Anfang Januar sogar die Gründung einer grass roots lobby „Organizing for America“" angekündigt (Pear 2008; Cillizza 2009); diese Versuche dürften jedoch mit der Zeit abebben oder in reguläre Lobbyarbeit übergehen. 
Auf jeden Fall werden sie Obama nicht am professionellen Regieren hindern, wie er mit einer für demokratische Präsidenten ungewohnt fokussierten transition unter Beweis gestellt hat: Schon seine Rede am Wahlabend, in der er die Erwartungen seiner Anhänger dämpfte und sie auf einen „langen Weg“ und „steilen Anstieg“ einschwor (Obama 2008b), zeigte seine abgeklärte Fähigkeit zur strategischen Erwartungssteuerung und zum Krisenmanagement. Die Schnelligkeit, mit der Obama die zentralen Positionen im Weißen Haus besetzte und sein Kabinett vorstellte, hat man seit den Zeiten Carters, seit denen die transitions bis ins letzte Detail wissenschaftlich untersucht werden, noch nicht gesehen. Bemerkenswert war auch, dass er sich nicht scheute, seine Kontrahentin Hillary Clinton zur Außenministerin und manche ihrer Anhängerinnen sowie Gefolgsleute ihres Mannes für hohe Posten in der Administration vorzuschlagen. Er folgte hier - auch in der Nominierung so anerkannter Persönlichkeiten und Fachleute wie Tom Daschle (Gesundheit), Timothy Geithner (Finanzen), Lawrence Summers (Nationaler Wirtschaftsrat) oder Steven Chu (Energie) - erkennbar einem selbstbewussten ,team-ofrivals-approach" im Stile seines politischen Idols Abraham Lincoln (Goodwin 2006). Sein Festhalten an Bushs Verteidigungsminister Robert Gates und weiteren Republikanern im nationalen Sicherheitsapparat kann als vertrauensbildende Maßnahme gegenüber den Republikanern und dem Pentagon sowie als Signal dafür gewertet werden, dass er wirklich etwas erreichen will. Auch seine intensive Kontaktpflege zum Kongress und sein nicht weniger intensives socializing mit den Washington Insidern und dem nationalen Pressekorps (Leibovich 2009) zeigen, dass er die politikwissenschaftlichen Lektionen effizienter Regierungstechnik gelernt hat (Neustadt 1990).

Trotz seiner sorgfältigen Vorbereitung war Obama jedoch nicht vor personalpolitischen Rückschlägen gefeit: Nachdem schon frühzeitig sein erster Vorschlag für das Handelsministerium, New Mexicos Gouverneur Bill Richardson, aufgrund staatsanwaltlicher Ermittlungen wegen Korruption von seiner Kandidatur zurückgetreten war, konnte Obama auch den designierten Gesundheitsminister Tom Daschle nicht halten. Daschle zog seine Kandidatur Anfang Februar 2009 zurück, nachdem bekannt geworden war, dass er 128.000 Dollar an Steuern hinterzogen hatte (Baker 2009). Zuvor war schon ein anderer Star des Obama-Teams, Finanzminister Timothy Geithner, wegen ähnlicher Steuervergehen in die Kritik geraten, hatte aber die Bestätigung durch den Senat erhalten. Der jüngste Rückschlag für den neuen Präsidenten kam an einem Freitag, den dreizehnten (Februar), als auch sein neuer 
Vorschlag für das Amt des Gesundheitsministers, der Republikaner Judd Gregg, seine Kandidatur zurückzog (Kornblut/Shear 2009).

Die personalpolitischen Kalamitäten Obamas weisen auf zwei Probleme hin, die sich unmittelbar aus seinen ambitionierten Wahlkampfversprechen ergeben: Erstens wird ihm sein Versprechen, die Art und Weise des Regierens in Washington zu ändern, mithin der Anspruch, dass sich auch die Mitglieder seiner Administration einem strengen Moralkodex zu unterwerfen haben, immer wieder Fallen stellen. Und zweitens wird auch das von Obama angestrebte Miteinander von Demokraten und Republikanern nach der politischen Polarisierung der letzten Jahrzehnte für alle Beteiligten eine große Herausforderung darstellen - die zu den ohnehin großen Herausforderungen der Wirtschaftskrise noch hinzukommt. Es erscheint derzeit nur schwer vorstellbar, wie er seine ambitionierten politischen Vorhaben ohne ein going public (Kernell 2006) und ohne Ausnutzung der strategischen Vorteile, welche die bully pulpit des Präsidenten ihm zumindest kurzfristig bietet (Edwards 2003), durchsetzen könnte.

\section{Literatur}

Bai, Matt, 2008: Is Obama the End of Black Politics?, in: New York Times Magazine, 10.08.08, http://www.nytimes.com/2008/08/10/magazine/ 10politics-t.html (Stand: 13.02.09).

Baker, Peter, 2008: Choice of Palin is a Bold Move by McCain, With Risks, in: New York Times, 30.08.08, http://www.nytimes.com/2008/08/30/us/ politics/30assess.html (Stand: 01.09.08).

Baker, Peter, 2009: Obama's Pledge to Reform Ethics Faces Early Test, in:

New York Times, 03.02.09, http://www.nytimes.com/2009/02/03/us/ politics/03lobby.html (Stand: 13.02.09).

Balz, Dan/Kornblut, Anne E., 2007: Top Aides Leave McCain Camp, in: Washington Post, 11.07.07, S. A 1.

Barnes, James A., 2008: The Art of Wooing, in: National Journal 40 (8), 18-24. Barnes, James A./Cohen, Richard E., 2004: The Manana Vote, in: National Journal 36 (23), 1760-1767.

Black, Earl/Black, Merle, 2002: The Rise of Southern Republicans, Cambridge. Brinkbäumer, Klaus, 2009: Der Boss des Präsidenten, in: Der Spiegel, 05.01.09, S. 76-88. 
Brookings (Hrsg.), 2008: Transcripts of Five Panels on the 2008 Presidential Election (I: Political Parties and Partisanship: The American Electorate, 12.09.08; II: Election Fundamentals: The Economy, the War and the President, 26.09.08; III: Issues, Ideology, Gender, and Race in the 2008 Election, 17.10.08; IV: Campaign Effects in the 2008 Election: Money, Ads and Mobilization, 31.10.08; V: What the 2008 Election Meant: Politics and Government, 14.11.08), Washington, D.C., http://www.brookings. edu/topics/elections.aspx (Stand: 17.01.09).

Brownstein, Ronald, 2008: The First 21 $1^{\text {st }}$-Century Campaign, in: National Journal, 19.04.08, http://www.nationaljournal.com/njmagazine/cs_ 20080416 3324.php (Stand: 19.01.09).

Buell, Emmett H., 1996: The Invisible Primary, in: William G. Mayer (Hrsg.), In Pursuit of the White House, Chatham, 1-43.

Bumiller, Elisabeth, 2008: Internal Battles Divided McCain and Palin Camps, in: New York Times, 06.11.08, http://www.nytimes.com/2008/11/06/us/ politics/06mccain.html (Stand: 02.01.09).

Campbell, James E., 2001: The Referendum that didn't Happen: The Forecasts of the 2000 Presidential Election, in: PS: Political Science \& Politics 34, 33-38.

Campbell, James E., 2004: Introduction - The 2004 Presidential Election Forecasts, in: PS: Political Science \& Politics 37, 733-735.

Campbell, James E., 2008: Editor's Introduction: Forecasting the $2008 \mathrm{Na}-$ tional Elections, in: PS: Political Science \& Politics 41, 679-681, http:// www.apsanet.org/imgtest/PSOct08Campbell.pdf (Stand: 21.11.08).

Carter, Bill, 2008: Infomercial for Obama is Big Success in Ratings, in: New York Times, 31.10.08, http://www.nytimes.com/2008/10/31/us/politics/ 31rate.html (Stand: 17.01.09).

CFI (Campaign Finance Institute), 2008: Financing Presidential Elections - 2008 Presidential Campaign, http://www.cfinst.org/president/dataHistorical.aspx (Stand: 10.01.09).

Cillizza, Chris, 2009: Obama Announces Grass-Roots Lobby, in: Washington Post, 19.01.09, S. A8, http://www.washingtonpost.com/wp-dyn/content/article/2009/01/17/AR2009011703093.html (Stand: 19.01.09).

CNN, 2000: Voter Exit Polls, http://edition.cnn.com/ELECTION/2000/results/index.epolls.html (Stand: 29.12.08).

CNN, 2004: Voter Exit Polls, http://edition.cnn.com/ELECTION/2004/pages/results/states/US/P/00/epolls.0.html (Stand: 29.12.08). 
CNN, 2008: Voter Exit Polls, http://edition.cnn.com/ELECTION/2008/results/polls/ (Stand: 29.12.08).

Cohen, Marty u. a., 2008a: Political Parties in Rough Weather, in: The Forum 5 (4), Art. 3, http://www.bepress.com/forum/vol5/iss4/art3 (Stand: 21.11.08).

Cohen, Marty u. a., 2008b: The Invisible Primary in Presidential Nominations, 1980-2004, in: William G. Mayer (Hrsg.), The Making of the Presidential Candidates 2008, Lanham, 1-38.

Corrado, Anthony, 2005: Public Funding of Presidential Campaigns, in: ders./ Daniel R. Ortiz/Thomas E. Mann/Trevor Potter, The New Campaign Finance Sourcebook, Washington, D.C., 180-204.

Corrado, Anthony, 2006: Financing the 2004 Presidential General Election, in: David Magleby/Anthony Corrado/Kelly D. Patterson (Hrsg.), Financing the 2004 Election, Washington, D.C., 126-148.

Cost, Jay, 2008: How Obama Won the Nomination, in: Policy Review, Nr. 150, 59-74.

Davidson, Roger H./Oleszek, Walter J., 2006: Congress and Its Members, Washington, D.C.

DNCC (Democratic National Convention Committee), 2008: Renewing America's Promise. The 2008 Democratic National Platform, Denver, 25.08.08.

Edwards, George C. III, 2003: On Deaf Ears. The Limits of the Bully Pulpit, New Haven.

Edwards, George C. III, 2007: Governing by Campaigning. The Politics of the Bush Presidency, New York.

Edwards, George C. III/King, Desmond S. (Hrsg.), 2007: The Polarized Presidency of George W. Bush, New York.

FEC (Federal Election Commission), 2008: Presidential Campaign Finance, http://www.fec.gov/DisclosureSearch/MapAppRefreshCandList.do (Stand: 10.01.09)

Fiorina, Morris, 1981: Retrospective Voting in American National Elections, New Haven.

Fiorina, Morris/Abrams, Samuel J./Pope, Jeremy C., 2003: The 2000 US Presidential Election: Can Retrospective Voting Be Saved?, in: British Journal of Political Science 33, 163-187.

Fiorina, Morris/Abrams, Samuel J./Pope, Jeremy C., 2005: Culture War? The Myth of a Polarized America, New York. 
Flores, Henry, 2004: The 2004 WCVI National Latino Election Day Exit Poll, San Antonio, http://wcvi.org/latino_voter_research/polls/national/2004/flores.html (Stand: 29.12.08).

Freedman, Paul, 2006: Swift Boats and Tax Hikes: Campaign Advertising in the 2004 Election, in: Larry J. Sabato (Hrsg.), Divided States of America. The Slash and Burn Politics of the 2004 Presidential Election, New York, 165-178.

Gallup, 2008a: Daily Tracking Poll, http://www.gallup.com/poll/107674/Interactive-Graph-Follow-General-Election.aspx (Stand: 12.01.09).

Gallup, 2008b: Presidential Job Approval in Depth, http://www.gallup.com/ poll/1723/Presidential-Job-Approval-Depth.aspx (Stand: 31.12.08).

Goodwin, Doris Kearns, 2006: Team of Rivals. The Political Genius of Abraham Lincoln, New York.

Green, Joshua, 2008: The Front-Runner's Fall, in: The Atlantic Online, September, http://www.theatlantic.com/doc/200809/hillary-clinton-campaign (Stand: 21.11.08).

Greenblatt, Alan, 2008: Changing U.S. Electorate: Are Demographic Trends Reshaping U.S. Politics?, in: CQ Researcher 18 (20), 457-480.

Horst, Patrick, 2004: Das amerikanische Notstandsregime nach dem 11. September 2001: Eine ,wehrhafte Demokratie“ auf Abwegen?, in: Uwe Backes/Eckhard Jesse (Hrsg.), Jahrbuch Extremismus \& Demokratie 16, Baden-Baden, 59-81.

Horst, Patrick, 2005: Der neue Republikanische US-Kongress: Polarisiert, zentralisiert und nachgiebig gegenüber dem Präsidenten, in: ZParl 36, 680-699.

Horst, Patrick/Bretthauer, Kathrin, 2001: Wahlentscheidende Effekte von Wahlkämpfen? Zur Aussagekraft gängiger Erklärungen anhand in der ZParl publizierter Wahlanalysen, in: ZParl 32, 387-408.

Jacobson, Gary C., 1990: The Electoral Origins of Divided Government. Competition in U.S. House Elections, 1946-1988, Boulder.

Jacobson, Gary C., 2007a: A Divider, Not a Uniter. George W. Bush and the American People, New York.

Jacobson, Gary C., 2007b: Referendum: The 2006 Midterm Congressional Elections, in: Political Science Quarterly 122, 1-24.

Jones, Charles O., 1998: Passages to the Presidency. From Campaigning to Governing, Washington, D.C.

Jones, Charles O., 2007: The American Presidency. A Very Short Introduction, Oxford. 
Jones, Jeffrey M., 2008: Obama Viewed as Winner of Third Debate. Completes „Sweep“ of Three Debates for Obama, Gallup Poll, 17.10.08, http://www.gallup.com/poll/111256/Obama-Viewed-Winner-Third-Debate.aspx (Stand: 03.11.08)

Jost, Kenneth, 2008: Women in Politics: Does Gender Bias Hurt Female Candidates?, in: CQ Researcher 18 (12), 265-288.

Katel, Peter, 2008: Race and Politics: Will Skin Color Influence the Presidential Election?, in: CQ Researcher 18 (25), 577-600.

Kernell, Samuel, 2006: Going Public. New Strategies of Presidential Leadership, Washington, D.C. (4. Aufl.).

Key, V. O., Jr., 1966: The Responsible Electorate. Rationality in Presidential Voting, 1936-1960, Cambridge.

Kornblut, Anne E./Shear, Michael D., 2009: Gregg Withdraws As Commerce Pick, in: Washington Post, 13.02.09, http://www.washingtonpost. com/wp-dyn/content/article/2009/02/12/AR2009021204029.html (Stand: 13.02.09).

Leal, David L. u. a., 2005: The Latino Vote in the 2004 Election, in: PS: Political Science \& Politics 38, 41-49.

Leibovich, Mark, 2008: Between Obama and the Press, in: New York Times Magazine, 21.12.08, http://www.nytimes.com/2008/12/21Gibbs-t.html (Stand: 17.12.08).

Leibovich, Mark, 2009: The Making of a New Washington Insider, in: New York Times Magazine, 17.01.09, http://www.nytimes.com/2009/01/17/ us/politics/17memo.html (Stand: 17.01.09).

Liedhegener, Antonius, 2005: „J. F. K.“- Amerikanische Katholiken und die Präsidentschaftswahlen 1960 und 2004, in: Torsten Oppelland/Werner Kremp, (Hrsg.), Die USA im Wahljahr 2004, Trier, 135-162.

Lopez, Mark Hugo, 2008: The Hispanic Vote in the 2008 Election, Washington, D.C., Pew Hispanic Center, November.

Los Angeles Times, 1992: National Election Day Exit Poll, http://roperweb. ropercenter.uconn.edu/cgi-bin/hsrun.exe/Roperweb/Catalog40/StateId/ DPtA9m-ovY3LfLpabvRdi3tuZZxtG-48HD/HAHTpage/file?fi=26811 (Stand: 31.12.08).

McDonald, Michael, 2008: The United States Elections Project. Voter Turnout, http://elections.gmu.edu/voter_turnout.htm (Stand: 16.12.08).

Mayer, William G., 2008: Handicapping the 2008 Nomination Races: An Early Winter Prospectus, in: The Forum 5 (4), Art. 2, http://www.bepress. com/forum/vol5/iss4/art2 (Stand: 21.11.08). 
Minushkin, Susan/Lopez, Mark Hugo, 2008: The Hispanic Vote in the 2008 Democratic Presidential Primaries, Washington, D. C., Pew Hispanic Center, Juni.

Nagourney, Adam, 2008a: Internal Politics Heat Up at McCain Campaign, in: New York Times, 08.07.08, http://www.nytimes.com/2008/07/08/us/ politics/08mccain.html (Stand: 12.01.09).

Nagourney, Adam, 2008b: Candidates Make Their Final Push on Reshaped Map, in: New York Times, 02.11.08, http://www.nytimes.com/2008/11/02/ us/politics/02states.html (Stand: 03.11.08).

Nagourney, Adam/Rutenberg, Jim/Zeleny, Jeff, 2008: Near-Flawless Run Is Credited in Victory, in: New York Times, 5. November, http://www.nytimes.com/2008/11/05/us/politics/05recon.html (Stand: 12.01.09).

NCSL (National Conference of State Legislatures), 2008: StateVote 2008, http://www.ncsl.org/statevote/StateVote2008.htm (Stand: 22.11.08).

Neustadt, Richard E., 1990: Presidential Power and the Modern Presidents. The Politics of Leadership from Roosevelt to Reagan, New York.

New York Times, 2008: The Ad Campaign, http://video.nytimes.com/video/playlist/politics/the-ad-campaign/1194811622223/index.html (Stand: 16.01.09)

Obama, Barack, 2004: Dreams from my Father. A Story of Race and Inheritance, New York.

Obama, Barack, 2006: The Audacity of Hope. Thoughts on Reclaiming the American Dream, New York.

Obama, Barack, 2008a: Speech on Race, Philadelphia, 18.03.08, http://www. nytimes.com/2008/03/18/us/politics/18text-obama.html (Stand: 17.01.09).

Obama, Barack, 2008b: Victory Speech, Chicago, 04.11.08, http:/www.nytimes.com/2008/03/18/us/politics/18text-obama.html (Stand: 17.01.09).

Ornstein, Norman/Mann, Thomas E. (Hrsg.), 2000: The Permanent Campaign and Its Future, Washington, D. C.

Pear, Robert, 2008: At House Party on Health Care, the Diagnosis is It's Broken, in: Washington Post, 23.12.08, http://www.nytimes. com/2008/12/23/health/23health.html (Stand: 19.01.09).

Polsby, Nelson W./Wildavsky, Aaron, 2008: Presidential Elections. Strategies and Structures of American Politics, Lanham.

Roper Center of Public Opinion Research, 2008a: National Election Day Exit Polls, http://www.ropercenter.uconn.edu/elections/common/exitpolls.html (Stand: 31.12.08).

Roper Center of Public Opinion Research, 2008b: Presidential Approval, http://webapps.ropercenter.uconn.edu/CFIDE/roper/presidential/webroot/presidential_rating.cfm (Stand: 31.12 .08 ). 
Rüb, Matthias, 2008: Die Leiden der Nachfahren Christi in Illinois, in: FAZ, 25.03.08, S. 3.

Saslow, Eli, 2008: Democrats Registering in Record Numbers. One Million New Voters For Last Seven Primaries, in: Washington Post, 28.04.08, S. A 1, http://www.washingtonpost.com/wp-dyn/content/story/2008/04/27/ ST2008042702368.html (Stand: 29.04.08).

Shaw, Daron R., 2006: The Race to 270. The Electoral College and the Campaign Strategies of 2000 and 2004, Chicago.

Sinclair, Barbara, 2006: Party Wars. Polarization and the Politics of National Policy Making, Norman.

Storey, Tim/Smith, Edward, 2008: Election 2008: Making History, in: State Legislatures, Dezember, http://www.ncsl.org/magazine/articles/2008/ 08sldec08_makinghistory.pdf, 14-17 (Stand: 22.11.08).

Suro, Roberto/Fry, Richard/Passel, Jeffrey, 2005: Hispanics and the 2004 Election: Population, Electorate and Voters, Washington, D. C., Pew Hispanic Center, Juni.

Turner, Robert C., 2005: Do Nebraska and Maine Have the Right Idea? The Political and Partisan Implications of the District System, in: Presidential Studies Quarterly 35, 116-137.

U.S. Census Bureau, 2008: Statistical Abstract of the United States. Section 7: Elections, http://www.census.gov/prod/2007pubs/08abstract/election. pdf (Stand: 21.11.08).

Walker, Martin, 2008: The Year of the Insurgents: the 2008 US Presidential Campaign, in: International Affairs 84, 1095-1107.

Wlezien, Christopher, 2001: On Forecasting the Presidential Vote, in: PS: Political Science \& Politics 34, 25-31.

Zelizer, Julian E., 2008: Worst Campaign Ever?, in: Newsweek, 05.11.08, http://www.newsweek.com/id/167561 (Stand: 12.01.09).

Korrespondenzanschrift:

Dr. Patrick Horst

Krohnskamp $35 \mathrm{~g}$

22301 Hamburg

E-Mail: patrick-horst@t-online.de 